\title{
Impact of lithium pellets on the plasma performance in the ASDEX Upgrade all-metal-wall tokamak
}

\author{
P. T. Lang 1), R. Maingi 2), D.K. Mansfield 2), R.M. McDermott 1), R. Neu 1,3), \\ E. Wolfrum 1), R. Arredondo Parra 1), M. Bernert 1), G. Birkenmeier 1,3), A. Diallo 2), \\ M. Dunne 1), E. Fable 1), R. Fischer 1), B. Geiger 1), A. Hakola 4), V. Nikolaeva 5,1,3), \\ A. Kappatou 1), F. Laggner 1), M. Oberkofler 1), B. Ploeckl 1), S. Potzel 1), T. Pütterich 1), \\ B. Sieglin 1), T. Szepesi 6), ASDEX Upgrade Team \\ 1) MPI für Plasmaphysik, Boltzmannstr. 2, 85748 Garching, Germany \\ 2) Princeton Plasma Physics Laboratory, PO Box 451, Princeton, NJ 08543, USA \\ 3) Technische Universität München, Boltzmannstr. 15, 85748 Garching, Germany \\ 4) VTT Technical Research Centre of Finland Ltd., PO Box 1000, FI-02044 VTT, Finland \\ 5) Instituto de Plasmas e Fusão Nuclear, Instituto Superior Técnico, \\ Universidade Lisboa, Portugal \\ 6) Wigner RCP RMI, Konkoly Thege u. 29 - 33, H - 1121 Budapest, Hungary
}

\section{E-mail contact of main author: peter.lang@ipp.mpg.de}

\begin{abstract}
The impact of lithium (Li) on the plasma performance was investigated at the ASDEX Upgrade tokamak, which features a full tungsten wall. Li pellets containing $1.6 \times 10^{20} \mathrm{Li}$ atoms were launched with a speed of $600 \mathrm{~m} / \mathrm{s}$ to achieve a deep penetration into the plasma and minimize the impact on the first wall. Homogeneous transient Li concentrations in the plasma of up to $15 \%$ were established. The Li sustainment time in the plasma decreased with increasing heating power from 150 to $40 \mathrm{~ms}$. Due to the pellet rate being restricted to $2 \mathrm{~Hz}$ no Li pile up could take place. No significant positive impact on plasma properties as reported from other tokamak devices could be found; Li pellets rather caused a small reduction of plasma energy mainly due to enhanced radiation. Due to pellet injection, a short-lived Li layer was formed on plasma facing components, which lasted a few discharges and led to moderately beneficial effects during plasma start-up. Most pellets were found to trigger type-I ELMs, either by their direct local perturbation or indirectly by the altered edge conditions; however the reliability was less than $100 \%$.
\end{abstract}

\section{Introduction:}

The presence of $\mathrm{Li}$ in tokamak devices seems to have the potential both for significant performance enhancement by improving key plasma parameter and for mitigation of the harmful impact imposed by edge-localized modes (ELMs). In NSTX Li coating of the graphite plasma facing components (PFCs), applied either with pellets injected into helium discharges or by oven depositions between discharges, has been investigated. Benefits sometimes seen included a decrease in plasma density, inductive flux consumption, and ELM occurrence. Furthermore, increases in electron and ion temperature, energy confinement, and the occurrence of periods of edge and magneto-hydro-dynamic quiescence [1]. ELMs were eliminated by an inward shift of the density and pressure profiles away from the separatrix [2], and energy confinement improved continuously with the amount of Li deposition [3]. Continuous injection of a $\mathrm{Li}$ aerosol into the edge plasma in EAST resulted in steady high confinement (H-mode) mode phases without ELMs for a duration exceeding hundreds of energy confinement times [4]. Strong PFC coating employing daily amounts of $10-30 \mathrm{~g}$ using both powerful evaporative ovens and plasma injection of $\mathrm{Li}$ powder reduced also the impurity levels and improved significantly plasma stored energies and energy confinement 
times [5]. ELM-free H-mode phases with increased pedestal pressure and width were observed in DIII-D using graphite for its PFCs. A powder of $45 \mu \mathrm{m}$ diameter Li particles was injected from the vessel top by a dropper device consisting of a piezo-electric vibrating disk, releasing particles at a controlled rate. At a sufficient level transient ELM-free phases up to $350 \mathrm{~ms}$ occurred, showing enhanced pedestal electron density and temperature, resulting in more than a doubled electron pedestal pressure. Also, overall plasma pressure and energy confinement undergo a remarkable increase, the latter reaching an enhancement factor $\mathrm{H} 98_{\mathrm{y}, 2}$ [6] of 2, while it is about 1 in reference ELMing periods [7]. Recently, also robust ELM pacing was demonstrated injecting spherical sub-mm Li granules into ITER-like plasmas. Sustained enhancement of the ELM frequency by a factor of $3-5$ and a reduced peak ELM heat flux at the outer strike point was achieved [8].

Seemingly, Li could provide advantages for future high power fusion devices, such as ITER and DEMO, by improving plasma performance and/or mitigating the ELMs. However, this needs to be further tested under conditions relevant for a fusion reactor. In particular, so far confinement improvement was attributed either to wall conditioning effects or were of transient nature. Also, the claim for reliable ELM control has to be further substantiated by successful application under various conditions. ASDEX Upgrade (AUG) is currently the only reactor with an all-tungsten-wall and thus represents a key point in parameter space. Hence, it was decided to perform exploratory experiments in order to find out if beneficial effects can be observed also in AUG. In particular, the experiments targeted a Li deposition deep inside the plasma to minimize the conditioning effect on the first wall. To achieve this goal, the decision was made to revive a gas gun launcher capable of accelerating mm-size, room-temperature, solid-state pellets to speeds of several hundred $\mathrm{m} / \mathrm{s}$. It was reasonably straightforward to adapt the gun to Li-pellets while realizing useful operational parameters, namely pellet size, velocity, and deep plasma penetration. Admittedly, the repetition rate restriction to a few $\mathrm{Hz}$ required sufficiently large pellets in order to achieve an adequate $\mathrm{Li}$ particle injection rate. As an initial benchmark for the tailoring of the pellet size we took the optimum Li injection rate in the $10 \mathrm{mg} / \mathrm{s}$ range found in DIII-D when injecting $\mu$ g-sized Li granules at low speeds [7]. Though larger and faster pellets would result in a more efficient Li delivery into the core plasma, we rather aimed at an injection rate of only few $\mathrm{mg} / \mathrm{s}$.

After commissioning and characterising the Li-injection gun in the test bed, the entire launching system was installed and integrated into the AUG experiment before the 2015/16 campaign. Finally, commissioned and trimmed for reliable operation during the restart phase, $\mathrm{Li}$ pellet injection was performed parasitically as well as in a series of dedicated experiments during the first phase of the campaign. Investigations were focussed on the question of whether or not the presence of a significant amount of $\mathrm{Li}$ in the core plasma has a significant impact on the plasma performance. To protect the first wall and prevent a Li contamination of PFCs and other in vessel components, a maximum budget for Li injection into AUG of $1 \mathrm{~g}$ was set.

\section{Setup: ASDEX Upgrade, Li pellet launcher and diagnostics}

AUG is a medium-sized divertor tokamak (major radius $\mathrm{R}_{0}=1.65 \mathrm{~m}$, minor radius $\mathrm{a}_{0}=0.5 \mathrm{~m}$, plasma current $\mathrm{I}_{\mathrm{P}}$ of up to $1.4 \mathrm{MA}$, toroidal magnetic field $\mathrm{B}_{\mathrm{t}}$ of up to $3.1 \mathrm{~T}$ ). All PFCs are covered with tungsten (W). Investigations reported here were performed early in the 2015/16 campaign operating with the redesigned solid W divertor-III [9]. The versatile set of auxiliary heating systems comprises up to $20 \mathrm{MW}$ neutral beam injection (NI), up to $6 \mathrm{MW}$ ion 
cyclotron resonance heating (ICRH), and up to $5 \mathrm{MW}$ electron cyclotron resonance heating (ECRH). An interlock (ILK) system monitoring several key plasma parameters is employed for safety and machine protection; in the experiment presented here it was employed to prevent pellet injection under unfavourable plasma conditions or after a disruption. A detailed report on the Li pellet launcher system can be found elsewhere [10], only a brief description is provided here. A sketch of the injection system as it was installed at AUG is shown in figure 1.

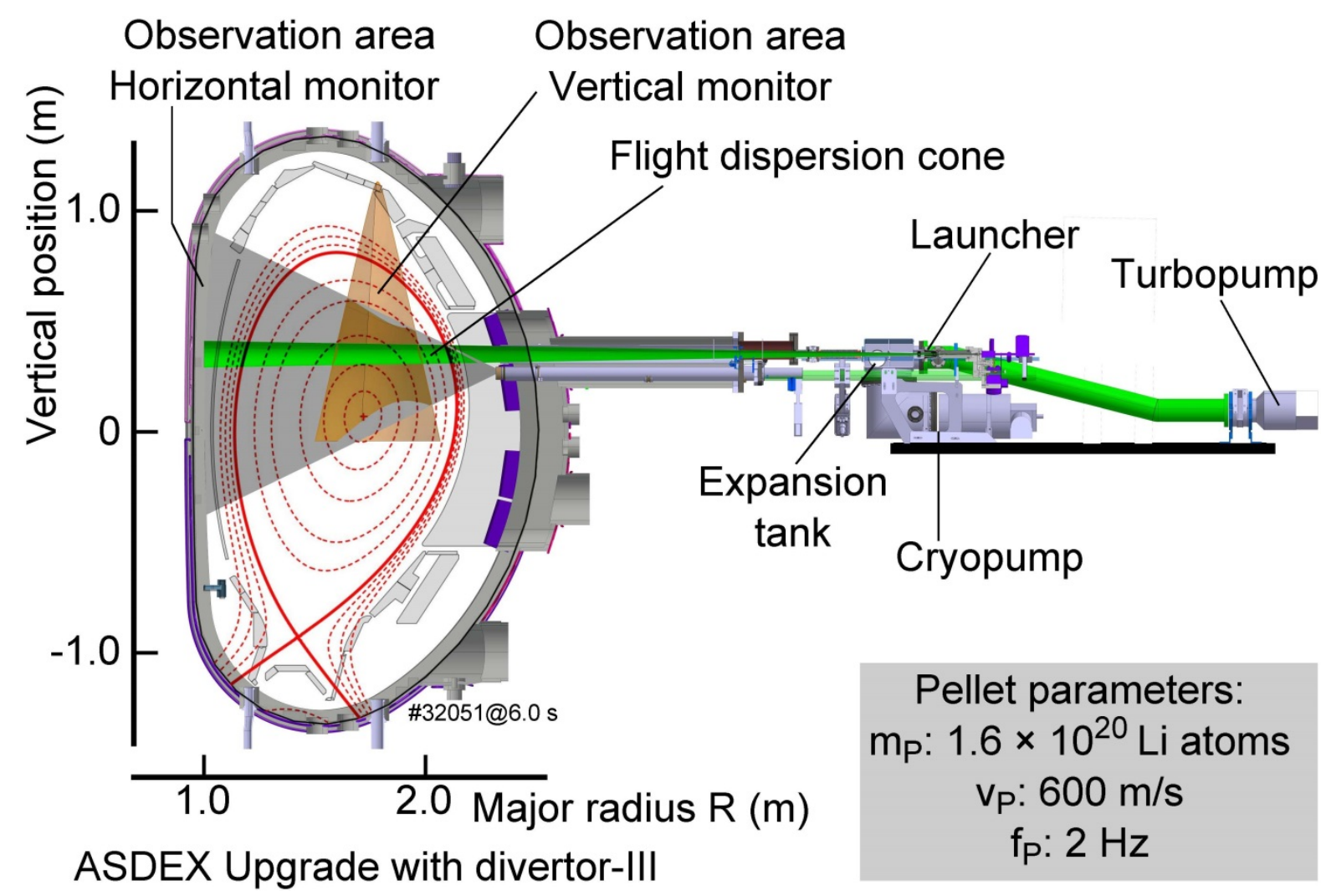

Figure 1: Setup of the Li pellet launching system and observation geometry of the pellet ablation monitors as installed at AUG during the first phase of the campaign 2015/16.

Our system can inject pellets containing nominally $1.64 \times 10^{20} \mathrm{Li}$ atoms $\left(\mathrm{m}_{\mathrm{P}} \approx 1.89 \mathrm{mg}\right)$ forming a pre-programmed train with a repetition rate up to a maximum of $2 \mathrm{~Hz}$. Its revolver plate magazine hosts up to 36 pellets stored in equally spaced holes of diameter $1.5 \mathrm{~mm}$, preloaded by mechanical double-stage extrusion of the soft Li originating from a $6 \mathrm{~mm}$ diameter wire. The pellet speed depends on the propellant gas pressure applied. To avoid undesired contamination of the plasma, deuterium (D), which is the most frequency used main ion in AUG plasmas, was chosen as propellant gas. Useful pressures range from 30 to 100 bar were found to result in pellet speeds of $420-700 \mathrm{~m} / \mathrm{s}$ for the applied acceleration geometry. With increasing propellant gas pressure the scattering angle of pellet trajectories was found to become smaller. Thus, a higher reliability for pellet delivery was achieved as more pellets arrive within the narrow acceptance cone for free flight to the plasma prescribed by the tight injection geometry. However, a higher gas pressure causes more gas flux. Finally, the best compromise was achieved for a pressure of 50 bar, which provided sufficient pellet delivery reliability, while keeping the resulting maximum parasitic flux to the torus vessel bearable. 
The resulting pellet speed of $585 \mathrm{~m} / \mathrm{s}$ (typical speeds scatter $32 \mathrm{~m} / \mathrm{s}$ ) turned out to be adequate to achieved sufficient plasma penetration. The main measures taken to limit the propellant gas load to the torus include the installation of a large expansion tank situated between the launcher and the torus entrance and enhanced pumping employing a strong cryo-pump. Even under maximum load conditions, when firing propellant pulses at $2 \mathrm{~Hz}$ through the empty magazine, the measured throughput to the torus of about $6 \times 10^{20} \mathrm{D} / \mathrm{s}$ remained below the set limit of $10^{21} \mathrm{D} / \mathrm{s}$. This value corresponds to a weak gas puff level for plasma operation and is, therefore, considered sufficiently low so as to not alter plasma behaviour.

Pellets have been injected via a horizontal port at $\mathrm{z}=0.35 \mathrm{~m}$ above the torus mid plane; this is typically $0.28 \mathrm{~m}$ above the plasma centre. For monitoring the Li pellet ablation radiation, two dedicated diodes were installed collecting all visible light emitted in the designated ablation region. One is observing the entire pellet path horizontally from behind while the other represents a top view covering the major part of the expected ablation region. The top view has a dynamic recording range adapted to the peak radiation and is hence capable of recording the entire visible ablation process at moderate sensitivity. In order to allow for a precise determination of the pellet's separatrix crossing the horizontal view was set to a very high sensitivity at the expense of saturation during the peak ablation. The pellet flight path with the dispersion cone angle achieved for the selected operational parameters and both monitoring areas are indicated in figure 1. For our investigations, we had the full versatile set of diagnostics available at AUG at our disposal for plasma characterisation and monitoring of the $\mathrm{Li}$ impact. Some dedicated efforts were taken to adapt or extend some of these diagnostics for $\mathrm{Li}$ pellet related measurements. For example, one of the standard video cameras was relocated close to the horizontal diode position at the injection access flange, spectrometers were set to record relevant spectral ranges and charge exchange recombination spectroscopy codes (CXRS) employed to obtain radial impurity density profiles were expanded to include Li.

The CXRS diagnostic used to measure the Li densities in the plasma is situated toroidally closely to the location of the Li-pellet injection, but the lines of sight do not intersect the pellet trajectory. The spectral line at $516.67 \mathrm{~nm}$ attributed to the transition $\mathrm{Li}^{++} \mathrm{n}=7 \rightarrow \mathrm{n}=5$ was used for the measurement and the atomic data for the analysis was taken from the ADAS database [11]. An uncertainty of $15 \%$ was assumed on all of the effective rates and included in the analysis of the $\mathrm{Li}$ densities. The $\mathrm{n}=2$ beam-impact effective rates were used as an estimate for the $n=2$ thermal charge exchange rates, though this contribution to the measured intensity was found to be less than $5 \%$ of the total signal. The plume contribution to this spectral line was also estimated and found to be on the order of $3 \%$ or less and was, therefore, not taken into account in the analysis. The charge exchange with the ground state, first energy component of the beam $(60 \mathrm{keV})$ comprised more than $80 \%$ of the signal. The effective rates used for this component were derived from cross-section data obtained using the ADAS universal formula, which should be reliable at these high beam energies. The perturbation of the Li pellet to the plasma density, temperature, and also on $\mathrm{Z}_{\text {eff }}$ were found to have a considerable impact on the calculation of the neutral beam attenuation and, therefore, care had to be taken to model the time evolution of these parameters correctly. Taking all of these things into account, the confidence in the Li-densities derived from the measured CXRS spectra is quite high.

An example of the successful launch and detection of a pellet train performed during the system commissioning phase is shown in figure 2. The first five pellets of a pre-programmed sequence were launched at a rate of $2 \mathrm{~Hz}$ into a steady phase with almost constant heating power; the last two pellets were blocked by the ILK system due to a recognized plasma 
disruption. Every pellet is clearly visible on both ablation monitors while their impact on the plasma density becomes obvious in the evolution of the line integrated density

$\overline{n_{e}} l=\int n_{e} d l,\left[\mathrm{~m}^{-2}\right]$ as measured by the laser interferometer. The sensitive horizontal pellet monitor also shows the propellant impinge on the plasma edge.

During the entire Li pellet campaign about 100 attempts to launch pellets were made, with $80 \%$ success rate; the remainder were found still sticking in the magazine. This is in total only about $150 \mathrm{mg}$ of Li launched into the vessel, far below the $1 \mathrm{~g}$ set level imposed for the entire investigation.

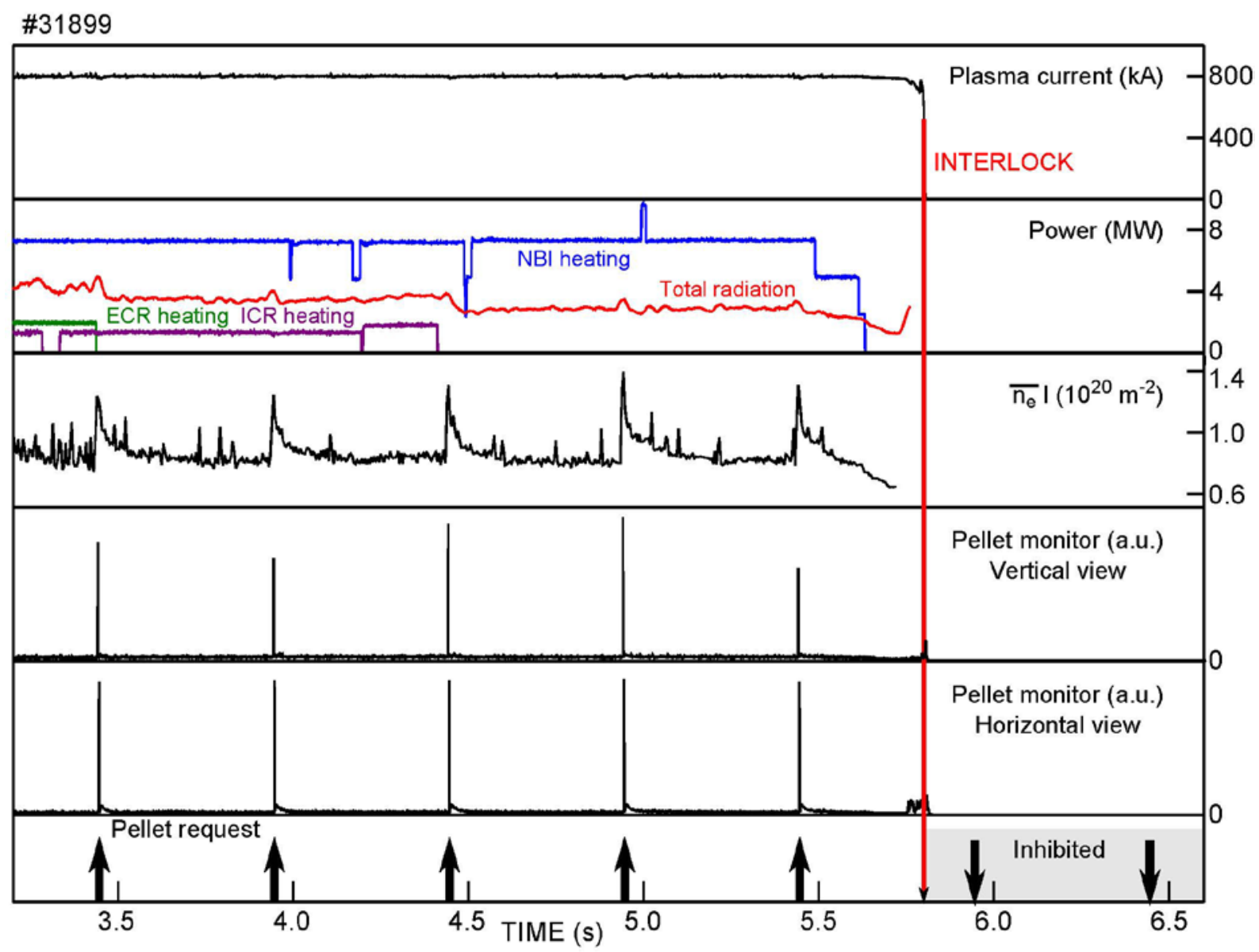

Figure 2: Demonstration of reliable Li pellet injector operation within the AUG safety system. The pre-programmed pellet sequence (7 pellets at 2Hz) is stopped by the ILK caused by a disruption.

\section{Efficient Li deposition in the core plasma}

\subsection{Deep penetration and deposition}

The arrival time of pellets into the plasma was confirmed independently by several different diagnostics. Since the observed maximum increase of $\overline{n_{e}} l$ is very close to the expected value of $4 \times 10^{19} / \mathrm{m}^{2}$ as for example shown in figure 2 , it can be concluded that most pellets do arrive with their full design mass. Also, time-of-flight speed measurements performed at the 
launcher exit proved pellets were injected with the expected velocity. Together, these parameters resulted in reliable deep pellet penetration into the plasma. A typical example is presented in figure 3. The pellet shown here arrived with a velocity of $585 \mathrm{~m} / \mathrm{s}$. Taking the duration of the monitored radiation, pellet ablation lasts for $1.25 \mathrm{~ms}$. Assuming a flight along the designated paths at constant speed yields a penetration depths of $0.73 \mathrm{~m}$. The corresponding ablation and presumed initial deposition region is marked by an arrow in the insert of figure 3. Assumptions made for this simple mapping seem to be reasonable as confirmed by the strong reduction of ablation radiation coinciding with the pellet trajectory becoming tangential to the plasma flux surfaces. At this position the heat reservoir of the flux surface becomes exhausted due to the long pellet residence time and hence the rampant plasma shielding effect reduced the heat flux into the ablation cloud causing the drastic observed reduction of the ablation [12].

Ablation monitor signal (a.u.)

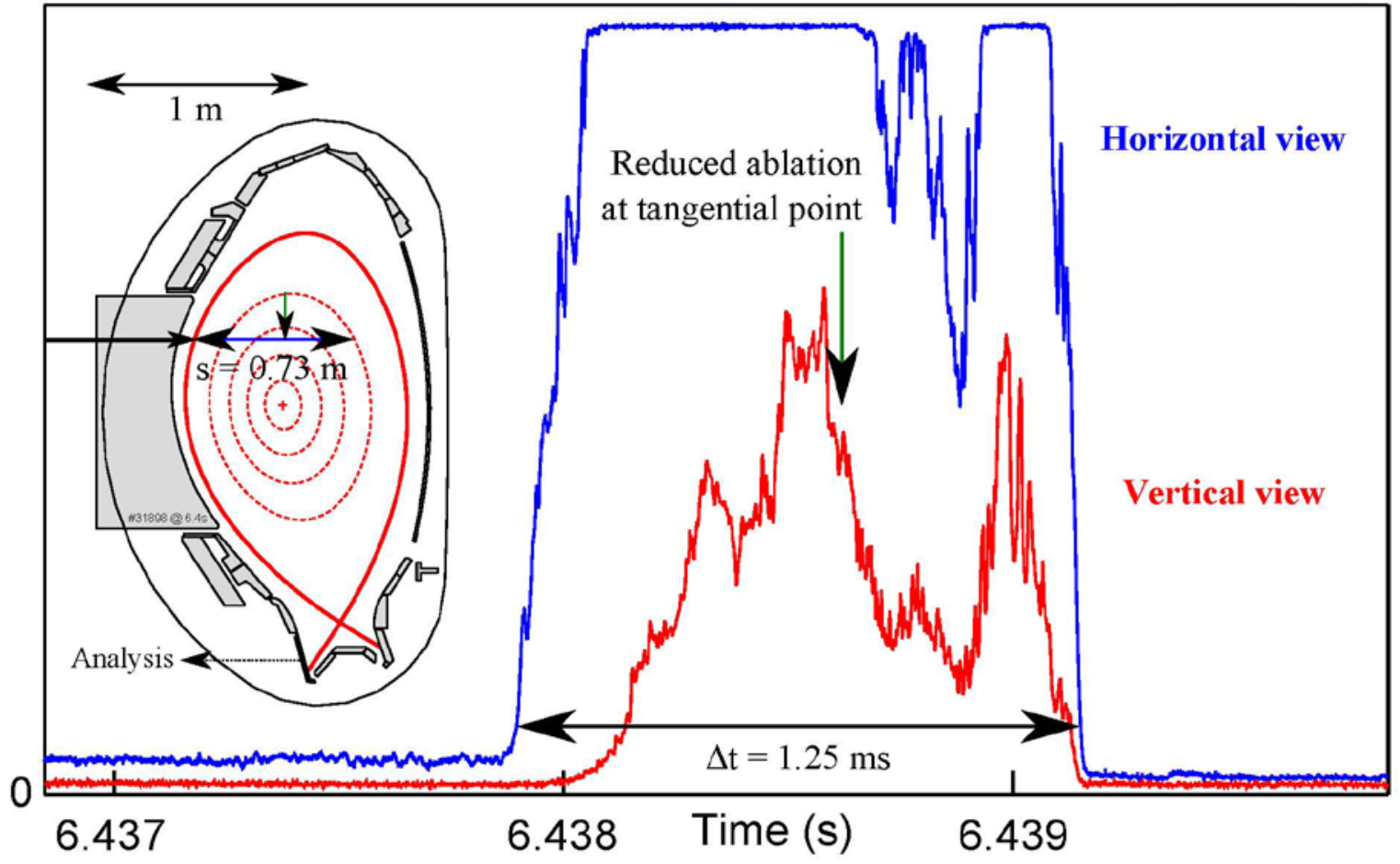

Figure 3: Example of a deeply penetrating Li pellet. Temporal evolution of Li pellet ablation radiation recorded by the sensitive horizontally viewing diode and the survey vertical view. Once the pellet trajectory becomes tangential to the flux surfaces, the strong local cooling of the plasma causes a sharp drop in radiation and presumably the ablation rate. Insert: Cross section of AUG with designated pellet path and mapped ablation region for a pellet speed assumed remaining at launch value of $585 \mathrm{~m} / \mathrm{s}$. The tile removed by the divertor manipulator for analysis is indicated as well.

Arrival of Li in the hot core plasma was detected after each successful injection of a pellet by the CXRS systems viewing a radially injected NBI. Within the beam volume, $\mathrm{Li}^{++}$is generated by charge exchange between fully stripped $\mathrm{Li}^{3+}$ and the injected $\mathrm{D}$ atoms. The $\mathrm{Li}^{++}$ ions then de-excite and emit characteristic line radiation, which can be monitored and used to obtain Li density profiles and time histories of the Li density at multiple radial locations. For the purpose of providing a simple "core Li-monitor", emission from the temperature 
broadened wing of the $\mathrm{n}=7-5 \mathrm{CX}$ line at $516.67 \mathrm{~nm}$ was only used as indicated in figure 4 . Using only this region removed the need to 'fit out' the passive Li emission, which originates from the Li diagnostics beam injected into the cold plasma edge.

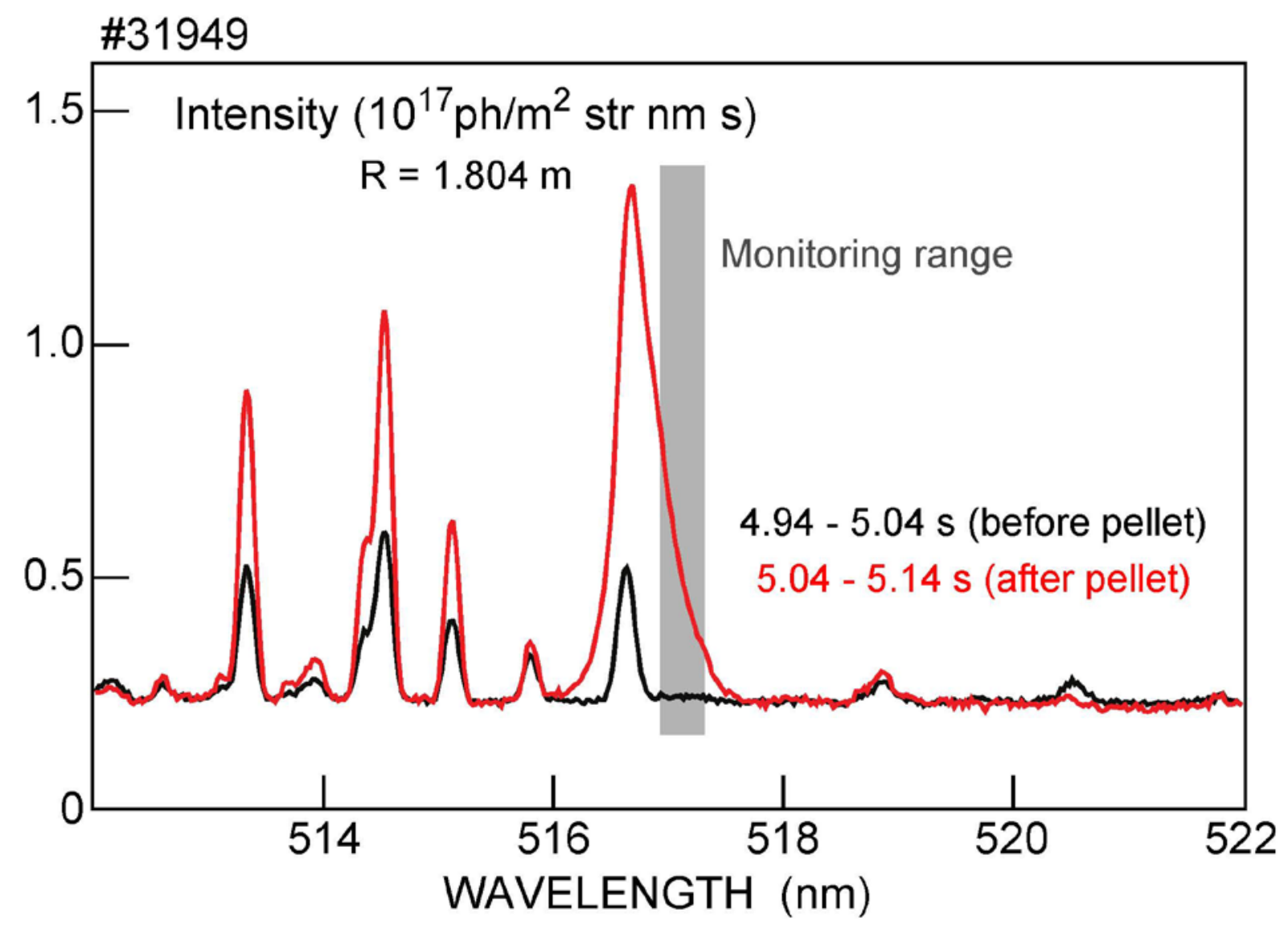

Figure 4: Radiation intensity recorded in the spectral region from 512 to $522 \mathrm{~nm}$ at the radial position $R=1.804 \mathrm{~m}$ immediately before (black) and just after injection of a Li pellet (red). The spectral range indicated by the grey bar covers emission from hot $\mathrm{Li}^{++}$ions only and is used to monitor the spatial and temporal of the Li as shown in figure 5.

The hot $\mathrm{Li}^{++}$monitoring signal recorded during the injection of a pellet train launched at $2 \mathrm{~Hz}$ is shown in figure 5 . The pellet sequence was intended to be comprised of 5 pellets. However, only the last 4 arrived intact. There is strong presence of $\mathrm{Li}$ in the entire accessible radial range following each successful pellet launch. A faint signal is observed at the misfired pellet time, indicating there is still some small amount of $\mathrm{Li}$ introduced to the plasma. It is also clearly visible that the $\mathrm{Li}$ presence is lasting less than $500 \mathrm{~ms}$ and thus, due to the maximum repetition rate of $2 \mathrm{~Hz}$, there is no pile up of $\mathrm{Li}$ in the plasma taking place. Hence, every single pellet can be considered and analysed as an individual event. 


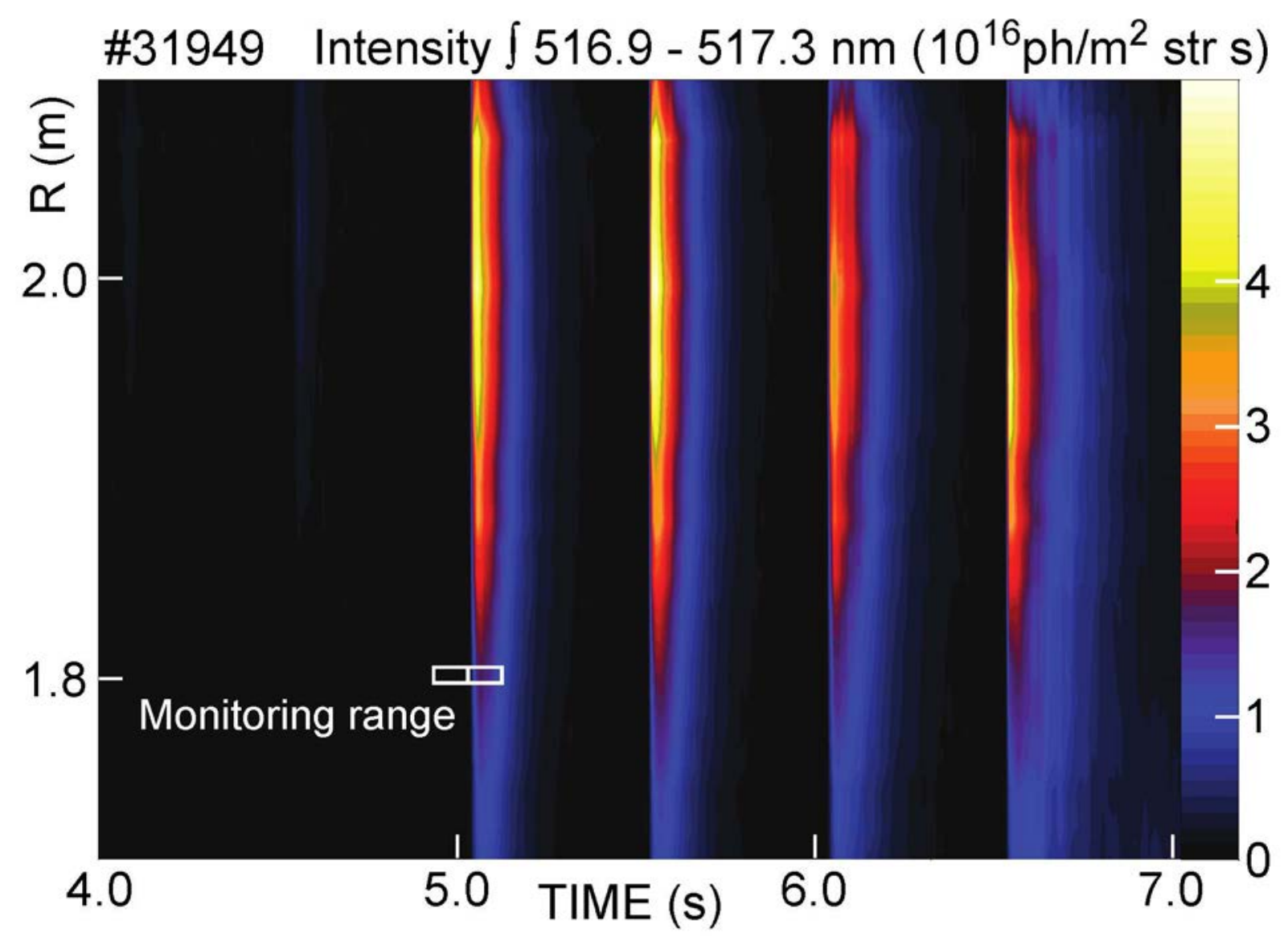

Figure 5: Temporal evolution of radiation emitted within the spectral range indicated in figure 4 versus major radius $R$. The white boxes indicate radial position and time frame selected for recording the full spectra shown in figure 4. Strong emission from hot $\mathrm{Li}^{++}$is observed after each successful injection of a Li pellet; for the first failed attempt only faint emission is observed likely caused by small pellet fragments.

\subsection{Homogeneous transient high $\mathrm{Li}$ concentration in the plasma}

In order to study the impact of $\mathrm{Li}$ on the plasma confinement, a sufficiently high and homogenous distribution of Li needed to be established and maintained for at least one energy confinement time. The Li-pellet injection system was, therefore, aiming to achieve a $\mathrm{Li}$ concentration of $0.1\left(\mathrm{c}_{\mathrm{Li}} \equiv \mathrm{n}_{\mathrm{Li}} / \mathrm{n}_{\mathrm{e}}\right.$; with $\mathrm{n}_{\mathrm{Li}}$ and $\mathrm{n}_{\mathrm{e}}$ the local densities of $\mathrm{Li}$ and electrons, respectively) and to maintain a significant concentration for about $0.1 \mathrm{~s}$. The target concentration was attained, as can be seen from figure 6, which shows results for a typical successfully injected pellet. The lines in the upper part of figure 6 display radial electron density profiles reconstructed by integrated data analysis (IDA) [13] at different times with respect to the pellet injection time $t_{0}$. The uncertainty level is in the range of $2 \times 10^{19} \mathrm{~m}^{-3}$. Corresponding profiles of Li densities as delivered by the CXRS diagnostics are shown in the lower part of figure 6 . Taking the pellet induced $\mathrm{Li}$ increase $12 \mathrm{~ms}$ after injection and calculating the resulting expected $\mathrm{n}_{\mathrm{e}}$ by assuming full $\mathrm{Li}$ ionisation yields the profile represented by the full dots. It agrees quite well with the corresponding IDA profiles. During its peak phase, $\mathrm{C}_{\mathrm{Li}}$ reaches approximately 0.15 . For the Li profile obtained $12 \mathrm{~ms}$ after the pellet arrival, the effect of initial pellet penetration (indicated by the arrow) and deposition 
remains visible. However, already at about 30 ms a nearly homogeneous doping across the entire plasma column is achieved with the Li profile becoming similar to the electron density $\mathrm{n}_{\mathrm{e}}$ profile. The homogeneous high Li concentration is decaying for the case shown with a time constant of approximately $100 \mathrm{~ms}$.

This can be seen more precisely from the temporal evolution of individual CXRS observation channels. Two of them, one observing the plasma core and the other plasma edge, are plotted in figure 7. Once the Li distribution has become homogeneous, the intensity decay (dots represent integrated intensity over a short time interval) fits very well to an exponential decay with a time constant $\tau$ of $100 \mathrm{~ms}$ (grey solid lines). The same $\tau$ value is observed for the decay of the Li induced electron density surplus, here represented by the line integrated density along the central chord of the DCN laser interferometer. Taking into account the modulation caused by the intermittent ELMs, also the monitor signal of the diagnostics Li beam shows the same behaviour. As a simple and robust signal for ELM monitoring, we employed the (thermo-) electric current into an outer divertor tile, measured as the voltage at a shunt resistor embedded in the tile mounting [14].

On the time scale relevant for this analysis, the Li can be considered distributed uniformly on flux surfaces. Homogenization of the pellet induced perturbation by parallel expansion is achieved typically in less than $1 \mathrm{~ms}$ [15] even faster than full ablation. Moreover, no indications were found for remnant poloidal/toroidal asymmetries appearing, due to the plasma rotation, as periodic variations in the $\mathrm{kHz}$ range.

The impact of ELMs has to be taken into account when considering the impact on the confinement. The uppermost box of figure 7 shows the evolution of the plasma stored energy, distinctly modulated by the ELM induced losses. Following the arrival of the Li pellet, there is a weak smooth transient reduction of the energy overlaid. As indicated, the maximum pellet induced change of plasma energy $\Delta \mathrm{W}$ emerges after about $50 \mathrm{~ms}$.

The observed evolution of the Li distribution during the decay phase was found to be in very good agreement with dedicated modelling of the evolution of an impurity blob as it diffuses inside the confinement region in a typical H-mode plasma [16] by a combination of the ASTRA transport modelling system [17] complemented with the TGLF module [18]. 


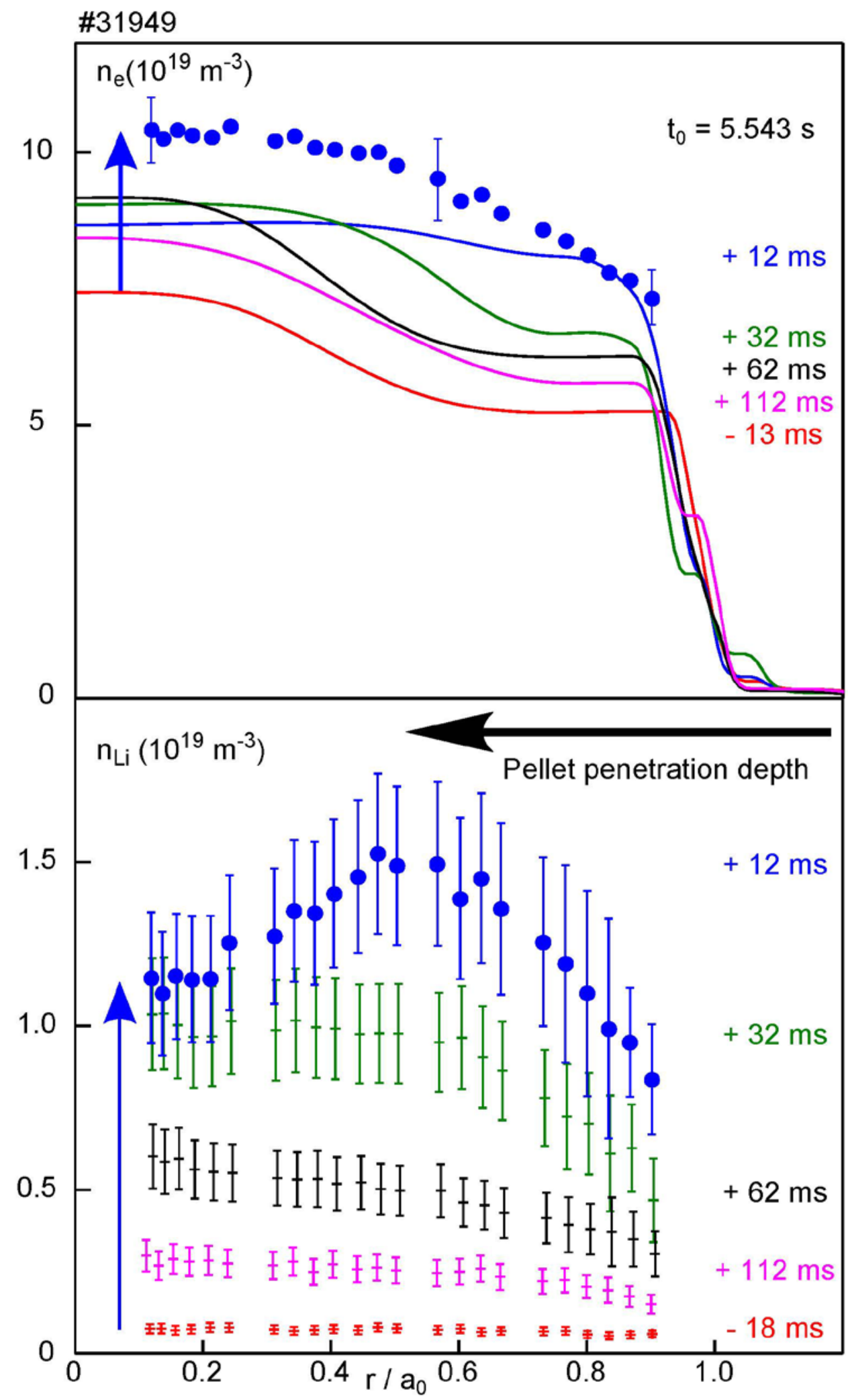

Figure 6: Temporal evolution of reconstructed electron (upper box, lines) and measured Li density profiles (lower box) before and after pellet injection. The electron density increase calculated $12 \mathrm{~ms}$ after the Li deposition (blue dots in upper part) fits well to the observed corresponding profile (blue solid line). 


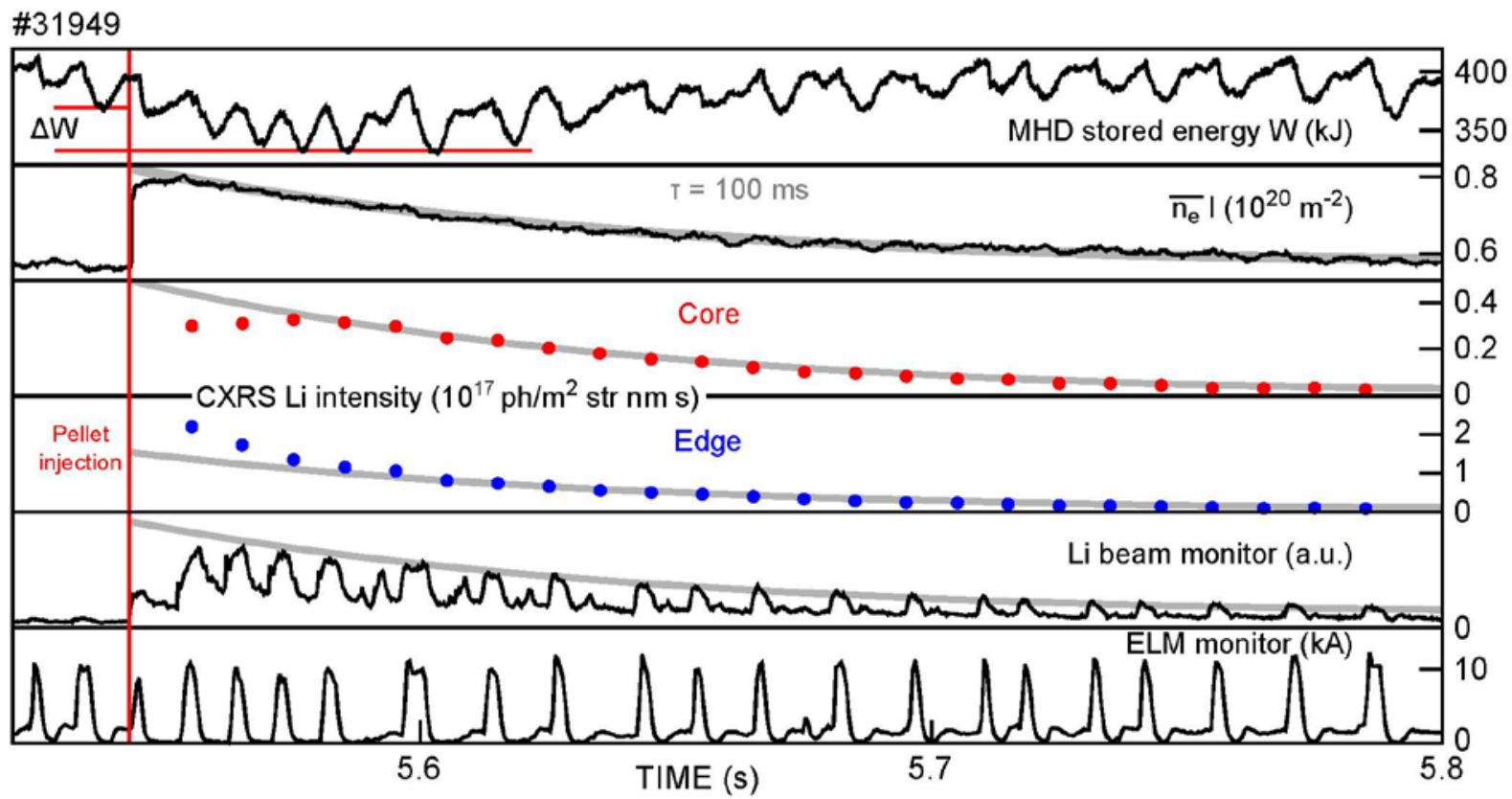

Figure 7: The Li concentration decays, for the case shown in figure 6 with a time constant of approximately $100 \mathrm{~ms}$. Almost identical sustainment times are obtained when analysing the decay of the entire $n_{e}$ inventory surplus or the Li radiation from different radial positions. For the analysis of the Li pellet's impact on the plasma energy (uppermost box) the modulation by ELM losses has to be taken into account.

\section{Effect of Li on the plasma performance}

\subsection{Monitoring and DIII-D reference discharges}

The essential question for this investigation was if the presence of $\mathrm{Li}$ in the core plasma can have a positive impact on key plasma parameters and improve confinement. Since AUG is one of only few devices with reactor relevant all-metal-PFCs, it was of particular interest how the plasma would react to Li under such conditions. Due to the strong ability of W to hamper plasma performance, operational conditions had to be adapted when changing from a carbon (C) to a full W wall in AUG, usually showing a trend to affect the confinement [19]. Seeding with nitrogen $(\mathrm{N})$ can recover such losses and restore conditions by adding only small impurity concentrations of about $2 \%$ [20]. A similar enhancement is found when adding $\mathrm{C}$ to the plasma by seeding with $\mathrm{CD}_{4}$ gas [20], while e.g. the injection of $\mathrm{He}$, simulating the presence of the fusion-produced ash, can lead to a clear reduction of the plasma stored energy both in non-seeded [21] and N-seeded discharges [22]. Recently, a region of high density localized in the scrape-off-layer at the high field side [23] was identified to play a major role for many of these effects in AUG. Influenced by e.g. gas fuelling or the presence of impurities, this region in turn impacts on the radial location of steep gradient in the edge pressure profile finally altering pedestal stability limits and hence the achievable plasma performance [24]. For a clear and comparable impact of $\mathrm{Li}$, we implemented a discharge 
configuration with a pronounced and reproducible sensitivity to such effects and a well documented impact to light impurities. In particular, long and steady $\mathrm{N}$ seeding phases at elevated confinement with respect to the non-seeded reference could be established. A direct comparison of $\mathrm{N}$ and $\mathrm{Li}$ impact was done, the temporal evolution of several crucial parameters for such a discharge is shown in the upper part of figure 8. For the steady main phase it is $\mathrm{I}_{\mathrm{P}}=1.0 \mathrm{MA}$; $\mathrm{B}_{\mathrm{t}}=2.47 \mathrm{~T}$; edge safety factor $\mathrm{q}_{95}=4.08$; plasma volume $\mathrm{V}_{\mathrm{p}}=12.2$ $\mathrm{m}^{3}$; elongation $\kappa=1.65$; upper and lower triangularity $\delta^{\mathrm{u}}=0.07$ and $\delta^{\mathrm{l}}=0.46$, respectively. Almost constant heating power was applied, including 10.0 MW NI and resonant core wave heating by 2.8 MW ICRH and 2.6 MW ECRH in order to prevent accumulation of impurities in the plasma centre [25]. Throughout, a persistent $D$ gas puffing rate of $\Gamma=2 \times 10^{22} \mathrm{D} / \mathrm{s}$ was set; this is at least 30 times the residual propellant gas flow for the Li pellet injector. A strong $\mathrm{N}$ puff was added for a fixed duration of $1.5 \mathrm{~s}$. As expected the plasma stored energy gets enhanced, finally reaching a magnitude even slightly above the value predicted by the presently widely used H-mode scaling IPB98(y,2) [6]. In parallel, ELM frequency and the total radiated power increase, too. In addition a strong drop of the of the divertor temperature from $40 \mathrm{eV}$ to $20 \mathrm{eV}$ and a modest increase of the divertor neutral gas pressure from 3 to $4 \mathrm{x}$ $10^{20} / \mathrm{m}^{3}$ takes place (data not shown in figure 8). From the attempt to inject five pellets, two arrive successfully in the plasma as can be deduced from the evolution of $\overline{n_{e}} l$ (data taken from the $\mathrm{CO}_{2}$ laser interferometer, calibrated to the DCN interferometer earlier in the discharge before data are lost due to fringe jumps). The propellant gas arriving for misfired pellets shows up in the pellet ablation monitor when impinging on the plasma edge region, but does not cause any noticeable impact on the key plasma parameters. One pellet arrives each in the $\mathrm{N}$-seeded and non-seeded phase. While the latter one seemingly arrives as one sound body, the first one has split into a large main body accompanied by a smaller fragment. This can be concluded from the wide angle video frames taken from behind with an integration time of 10 ms, shown in the lower part of figure 8 . The bright ablation zone for every pellet or pellet part is clearly visible; also the Li spreading out along the field lines shows up by the characteristic green radiation, contrasting to the red colored radiation stemming from neutral $\mathrm{D}$ in the vicinity of the last closed flux surface or in the divertor. For the Li pellet arriving in the nonseeding phase, no significant impact on the plasma stored energy can be recognized. For the $\mathrm{N}$-seeding case a short but distinct reduction of plasma stored energy takes place during the presence time of $\mathrm{Li}$, retracting about one quarter of the $\mathrm{N}$ induced enhancement. 

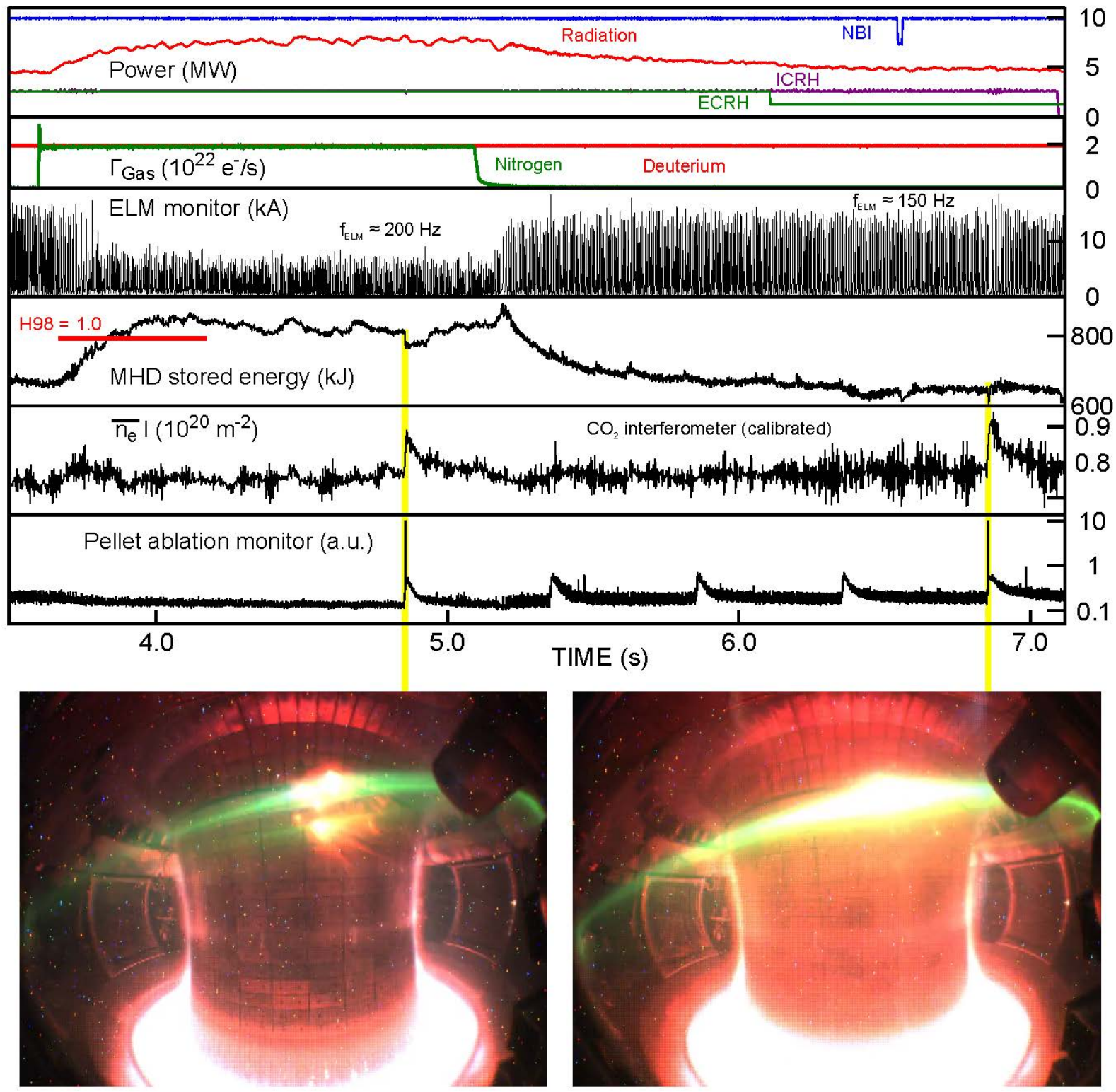

Figure 8: Upper: Key parameter evolution for a monitoring plasma discharge comprising a $\mathrm{N}$-seeding and a non-seeding phase. 5 attempts for a Li pellet launch delivered one successful pellet into each phase, clearly indicated by the impact on the density. Lower: Wide angle view video frame taken during arrival of the according pellets (indicated by yellow bars). Green clouds visualize the Li spreading along field lines. Neutral D from cooler plasma regions appears red. Clearly visible are the inner heat shield covered by tiles, the divertor region and several limiter structures at the outboard.

Additional examples of pellets injected into monitoring discharge are shown in figure 9, displaying an entirely non-seeded case in the upper and a phase with steady $\mathrm{N}$ puffing in the lower part. For both cases the plasmas parameters are essentially identical to those of discharge \#32056 shown in figure 8. Again, pellet arrival is monitored by the impact on $\overline{n_{e}} l$ (here the calibrated signal from the Bremsstrahlung was taken due to both laser 
interferometers signals becoming perturbed earlier in the discharge). There are two sound pellets in the non-seeded case; one misfired pellet is also indicated by the ablation monitor. In the N-seeding case, two sound pellets also arrive, and one misfire is detected by the ablation monitor. A fourth pellet showing up in the ablation monitor does not result in a measurable density increase; presumably this one was heavily fragmented and did not penetrate sufficiently. Henceforth, only pellets showing sufficient impact on $\overline{n_{e}} l$ and a sound appearance in the ablation monitor signal were regarded for further database analysis. The two corresponding pellets for each of the two cases shown in figure 9 thus confirmed the above findings. There is virtually no impact on the plasma stored energy in the non-seeding plasma, while there is a small but distinct negative impact in the $\mathrm{N}$ seeding case. To note all scales for both shots are identical, reflecting again the elevated plasma stored energy content arising from the $\mathrm{N}$ seeding.

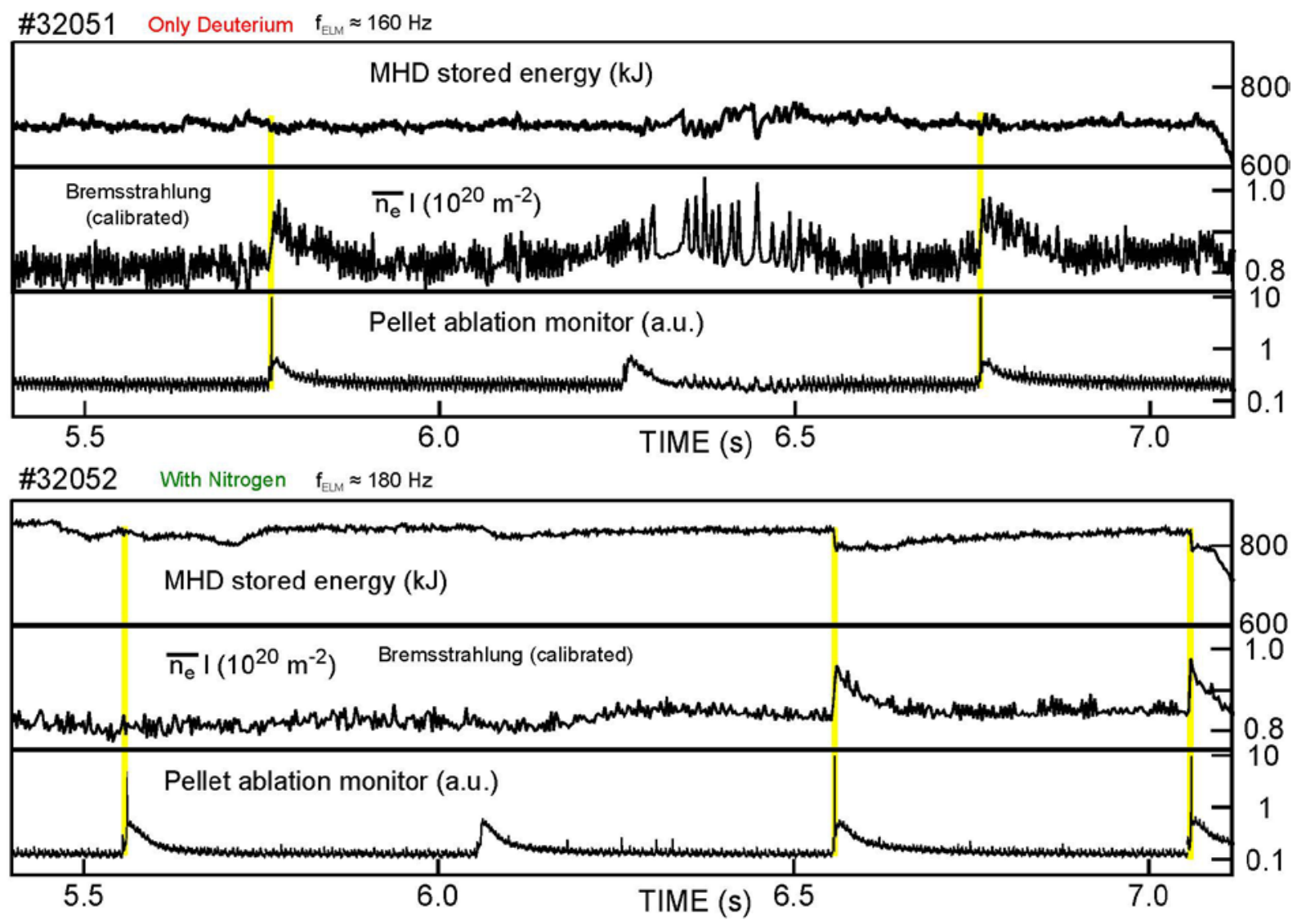

Figure 9: Monitoring plasmas without (upper) and with $N$ seeding (lower) with several pellet injection attempts. The pellet ablation monitor (note logarithmic scale) indicates also the propellant gas pulse. Not all ablation radiation spikes show a corresponding impact on the density, hinting at severe pellet fragmentation. Only the pellets reaching the plasma (indicated by vertical bars) are taken into account.

Before the optimized monitoring discharges were performed, first tests were made to check the impact of Li on plasma performance. After the first boronization of the campaign and still in the final commissioning phase of Li pellet launcher, this was done in discharges tailored for low density and collisionality like those in the DIII-D investigations [7]. These discharges had 
parameters $\mathrm{I}_{\mathrm{P}}=0.8 \mathrm{MA} ; \mathrm{B}_{\mathrm{t}}=2.48 \mathrm{~T} ; \mathrm{q}_{95}=5.3 ; \mathrm{V}_{\mathrm{p}}=13.0 \mathrm{~m}^{3} ; \kappa=1.65 ; \delta^{\mathrm{u}}=0.09$ and $\delta^{\mathrm{l}}=$ 0.41. ECRH was applied for core heating in addition to NBI; some gas was injected to suppress a too high $\mathrm{W}$ influx. Results are added to the database analysed in the next section. For this scenario, also one case with 1.2 MW ECRH, 4.9 MW NBI and $\Gamma=1 \times 10^{21} \mathrm{D} / \mathrm{s}$ was established closely matching the initial density and temperature pedestal conditions for a DIII-D reference experiment that showed a pronounced impact of Li granules dropped onto the plasma [shown in figure 15 of ref. 7]. Before application of Li granules the DIII-D pedestal top values were $\mathrm{n}_{\mathrm{e}}^{\text {ped }} \sim 3.5 \times 10^{19} \mathrm{~m}^{-3}$ and $\mathrm{T}_{\mathrm{e}}^{\text {ped }} \sim 500 \mathrm{eV}$. With $\mathrm{Li}$ application the prolonged appearance of the so-called bursty chirping mode led to an inward shift of the density profile, consequently also to an inward shift of the pressure profile, which resulted in a strongly improve pedestal stability. The improved DIII-D pedestal values reached $\mathrm{n}_{\mathrm{e}}{ }^{\text {ped }} \sim 4$ $\mathrm{x} 10^{19} \mathrm{~m}^{-3}$ and $\mathrm{T}_{\mathrm{e}}{ }^{\text {ped }} \sim 1.2 \mathrm{keV}$.

The closest match of AUG pedestals to the DIII-D pedestals before application of Li are shown in figure 10 in black with $\mathrm{n}_{\mathrm{e}}{ }^{\text {ped }} \sim 4 \times 10^{19} \mathrm{~m}^{-3}$ and $\mathrm{T}_{\mathrm{e}}{ }^{\text {ped }} \sim 500 \mathrm{eV}$. All profiles stem from IDA of Thomson scattering, ECE and DCN diagnostics [26]. They contain only interELM data and are averaged over 100 ms. Immediately after pellet injection, the density rises and the temperature drops (red profiles). The effect of the Li pellet is no longer evident within $300 \mathrm{~ms}$ (blue profiles). At all three time points shown in figure 10, the edge pressure profiles are very similar within the error bars, so we conclude that the edge stability is not changed. The bursty chirping mode was detected in DIII-D with the beam emission spectroscopy (BES) diagnostic but not in the magnetic diagnostics. At AUG there is no BES diagnostic with high temporal resolution installed. Instead the reflectometry diagnostic was set to fixed frequency, O-mode at $44 \mathrm{GHz}$, which corresponds to a cut off density of $2.4 \times 10^{19} \mathrm{~m}^{-3}$, located in the steep gradient region of the pedestal. 


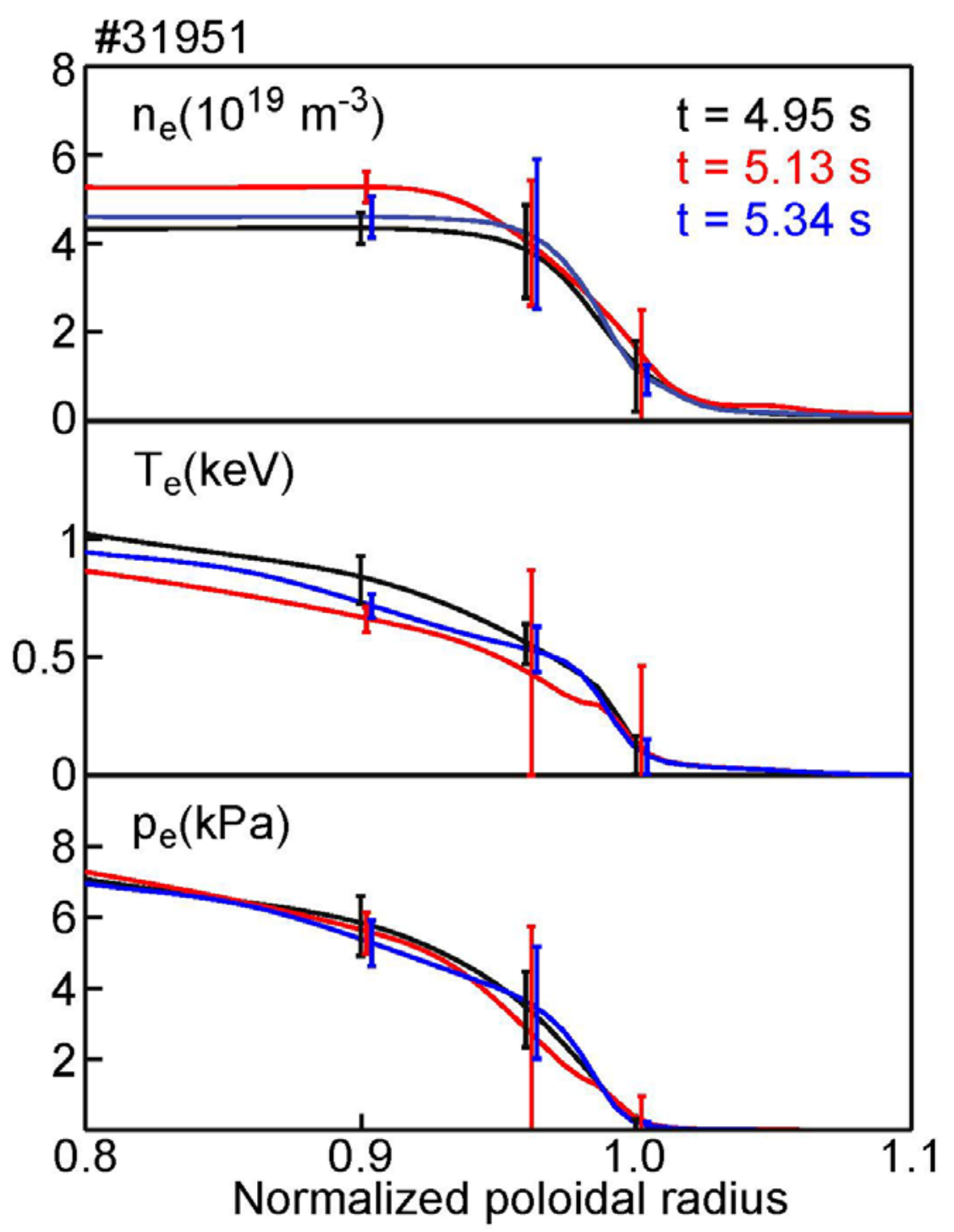

Figure 10: Electron density, temperature and pressure profiles (top to bottom) of the plasma edge region for a case closely matching the DIII-D reference case [shown in figure 15 of ref. 7]. Reference profile well before pellet injection ( $\mathrm{t}=4.95 \mathrm{~s}$ in black) and with just faded pellet impact ( $\mathrm{t}=5.34 \mathrm{~s}$ in blue) - as well as profiles close to maximum impact by pellet injection $(\mathrm{t}=5.13 \mathrm{~s}$ in red $)$.

The uppermost box of figure 11 shows a spectrogram of density fluctuations measured with O-mode reflectometry at $44 \mathrm{GHz}$. The integrated spectral power of 60 to $90 \mathrm{kHz}$ is shown as a time trace in the box below. The panel just below displays a time trace of the divertor thermo-current as ELM monitor and the pellet monitor signal. For comparison a spectrogram of a magnetic pick-up coil installed at the horizontal mid plane recording $\mathrm{dB}_{\mathrm{r}} / \mathrm{dt}$ is shown in the lowermost box. The Li pellet triggers an ELM at $\mathrm{t}=5.037 \mathrm{~s}$, and at $\mathrm{t}=5.042 \mathrm{~s}$ an increase of the density fluctuations between 60 and $90 \mathrm{kHz}$ can be observed. The same density fluctuation pattern can be observed in seven inter-ELM phases after pellet injection with decreasing intensity, corresponding to the decreasing Li content in the plasma. The phases occur in this frequency band after an ELM and fade away already before the next ELM. However, it is not clear, whether they do not exist anymore or the fluctuations move out of the observation range of the reflectometry. At the same time there is no activity in the magnetic 
spectrogram in this frequency range (see the red encircled regions with increased density fluctuations but little magnetic signal).
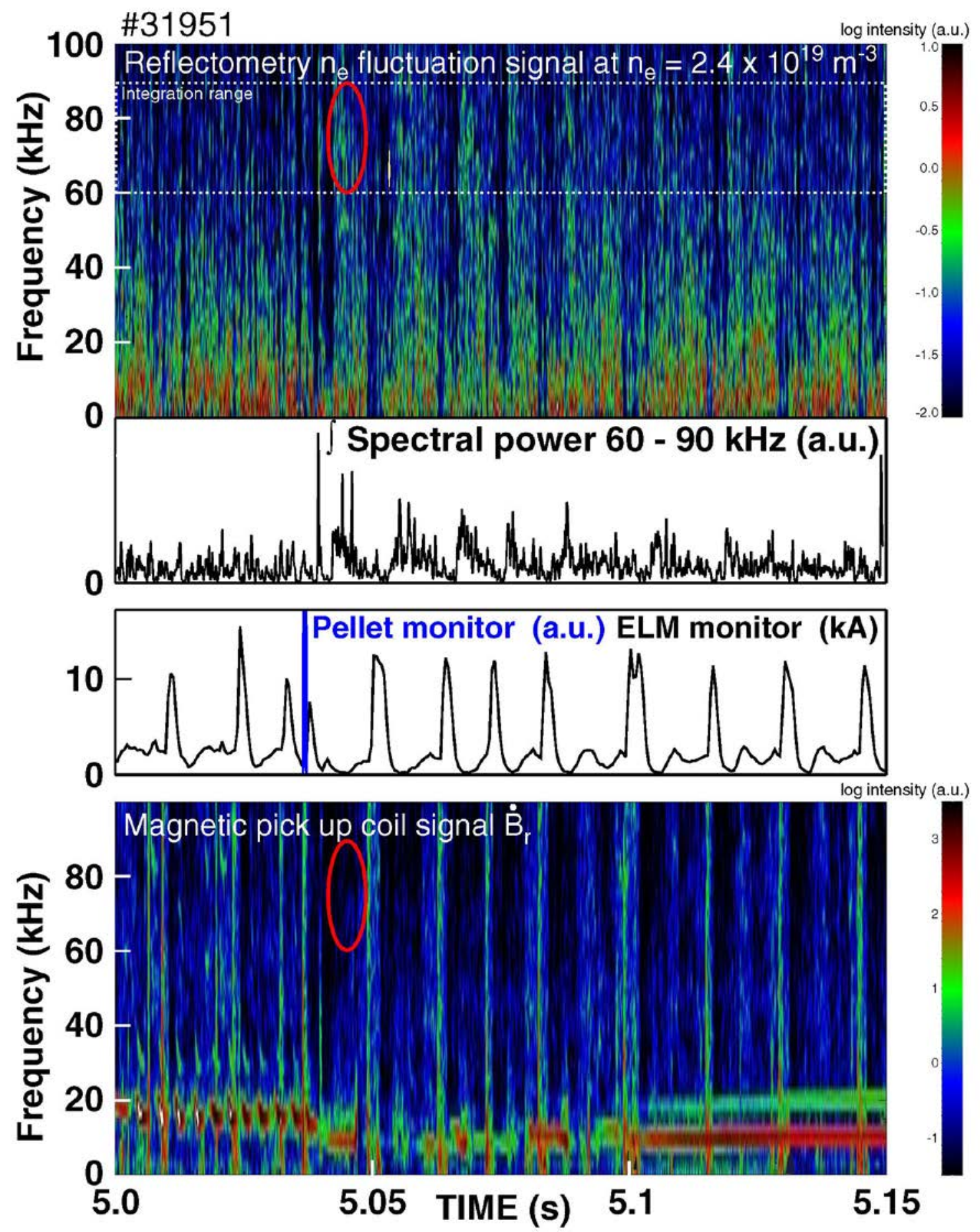

Figure 11: Top boxes: Spectrogram of density fluctuations from reflectometry at $n_{e}=2.410^{19}$ $\mathrm{m}^{-3}$ (upper) and time trace of spectral power integrated from $60-90 \mathrm{kHz}$ (lower). Middle box: time trace of ELM and pellet monitors. Bottom box: spectrogram of mid plane radial magnetic fluctuations. 
Summarising the observations, we can state that the Li-pellet triggers the occurrence of similar density fluctuations as in DIII-D, which are however not continuous and do not change the shape of the density profile sufficiently to give rise to improved pedestal stability.

\subsection{Li impact on energy confinement}

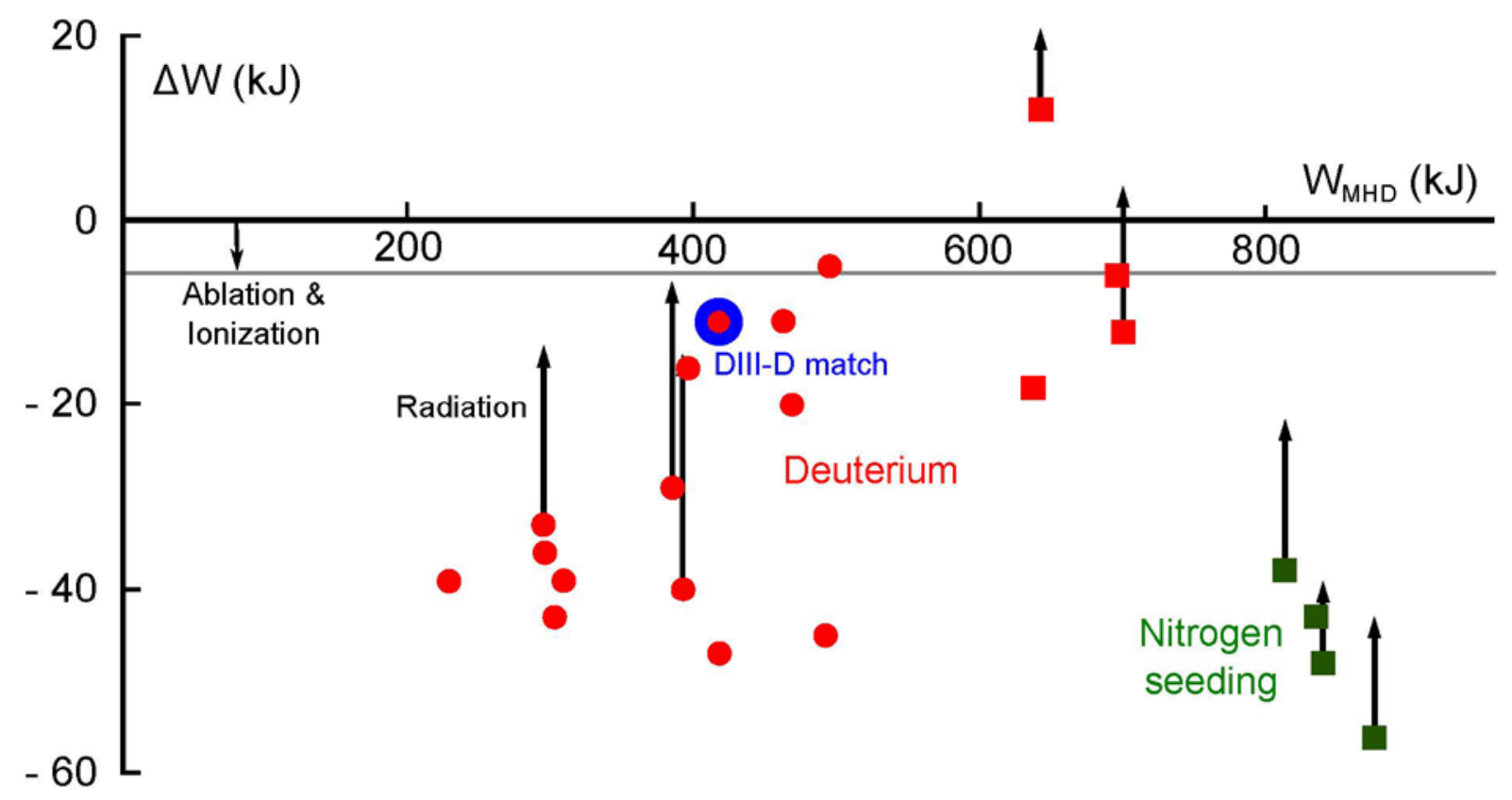

Figure 12: Pellet induced changes of the plasma energy versus initial plasma energy content. Dots: commissioning and piggy back experiments, DIII-D pedestal match indicated. Squares: monitoring experiments. Red: pure D plasmas, green: $N$ seeding applied. Grey line: energy consumption for ablation and ionisation. Upward arrows: radiative losses caused by $\mathrm{Li}$ pellet.

As described before, a database was compiled for all successful pellets comprising the monitoring and low density discharges, but also several piggy back experiments performed already during the restart phase mainly for launcher testing and commissioning. Hence, data cover a wide range of different plasma scenarios and also vessel conditions, since some tests were performed in the campaign's initial restart phase even before the first vessel boronization for wall conditioning took place. For the early experiments, the total applied heating power $\mathrm{P}_{\mathrm{h}}$ (sum of all applied auxiliary heating and Ohmic heating power) was gradually increased up to $12 \mathrm{MW}$ while the concluding monitoring discharges had all about $\mathrm{P}_{\mathrm{h}}=15 \mathrm{MW}$. All available data indicating the impact of Li on the plasma by $\Delta \mathrm{W}$ versus the plasma stored energy $\mathrm{W}_{\mathrm{MHD}}$ are plotted in figure 12. In order to eliminate the impact of the ELMs, data were taken by referring to the same phase in the ELM cycle, as indicated in figure 7. Red data points represent nominal pure D target plasmas; green ones $\mathrm{N}$ seeded discharges. Monitoring experiments are depicted by squares, all other by dots. With respect to $\mathrm{W}_{\mathrm{MHD}}$ there is a clear separation between the rest of the data and the monitoring experiments due to the separation by $\mathrm{P}_{\mathrm{h}}$. Non-seeded and $\mathrm{N}$ seeded monitoring are further separated at the $\mathrm{W}_{\mathrm{MHD}}$ axis as a consequence of the confinement enhancement effect in plasmas with virtually identical $\mathrm{P}_{\mathrm{h}}$. Here, also a clear separation with respect to the $\Delta \mathrm{W}$ values reflects the stronger $\mathrm{Li}$ impact in the $\mathrm{N}$ seeding cases. 
Altogether, injection of a Li pellet always resulted only in a small transient change of the plasma energy. No indication of a major impact on energy confinement was observed. With only one exception, the plasma energy was reduced only modestly. The D data seem to go well together, indicating the magnitude of this loss decreases with increasing plasma energy until it almost vanished for the discharges with the highest heating power. $\mathrm{N}$ seeding cases clearly deviate from the rest of the dataset.

To account for the observed plasma stored energy reduction, several potential loss processes were examined. First, the solid Li arriving in the plasma has to be heated up and evaporated, and the atoms fully ionized. Summing up all these losses yields an energy consumption per pellet of $5.4 \mathrm{~kJ}$. The horizontal solid grey line in figure 12 hence represents the true zero baseline for the impact of the deposited $\mathrm{Li}^{3+}$ ions. As can be seen in figure 2, some extra radiation is caused by the presence of the $\mathrm{Li}$. The total energy of this additional radiation was estimated from the fast XUV diode arrays measurements calibrated to the bolometer data for a couple of pellets; according values are displayed by the upward arrows in figure 12. Notably, tomographic reconstruction of the 2D radiation pattern could not identify regions of especially high radiation caused by the $\mathrm{Li}$; in particular no ring like structure e.g. close to the last closed flux surface could be identified. There are other minor effects caused by the Li altering the stored energy; as e.g. the fuelling induced cooling results in an increased resistivity causing an enhanced plasma loop voltage and hence Ohmic heating. However, such small magnitude effects cannot be quantified under time-varying ELMing plasma conditions. Taking into account radiation losses and the energy consumption for pellet ablation and ionization, in D plasmas the remaining impact on the confinement becomes marginal. For $\mathrm{W}_{\mathrm{MHD}}<400 \mathrm{~kJ}$ higher radiation losses are found compared to the monitoring plasmas and a slight net energy reduction takes place. In case of the non-seeding monitoring scenario there may be indication for a faint enhancement in the range of a few \%, but a significant confinement improvement by the Li can be ruled out.

In the $\mathrm{N}$ seeded monitoring discharges, losses of the $\mathrm{N}$ induced confinement surplus cannot be fully explained by radiation and pellet specific losses. We attribute this to the fuelling impact of the Li pellets. Notably, the same effect is observed when applying D pellets in plasma fuelling experiments. While no plasma energy loss takes place when fuelling to densities beyond the Greenwald density by $\mathrm{D}$ pellets in non-seeded plasma, a considerable fraction of the $\mathrm{N}$ induced confinement enhancement is reversed when combining pellet fuelling and $\mathrm{N}$ seeding [27]. This effect is obviously caused by a recurrent deleterious high field side high density region [24], since a significant increase of the according local density is also observed during the Li pellet induced density enhancement phase.

\subsection{Impact of heating power on Li sustainment time}

Higher radiation losses found under conditions with lower plasma stored energy and lower temperature can be partly explained by the strongly increasing cooling factor due to higher radiative power for Li below $300 \mathrm{eV}$ [28]. However, we attribute this behaviour mainly to the Li sustainment time in the plasma, which is found to increase with decreasing heating power and plasma stored energy. Sustainment times ( $\tau$ from exponential fit) are plotted versus $P_{h}$ in figure 13, derived from either the decay of the Li induced $\overline{n_{e}} l$ enhancement (open symbols) or the CXRS radiation from the edge (filled symbols). Colours and symbols correspond to those in figure 12. Li sustainment times decreasing from 150 to $40 \mathrm{~ms}$ show similar values and the same trend as the energy confinement time $\tau_{\mathrm{E}}$. $\tau_{\mathrm{E}}$ values approximated taking the ratio 
$\mathrm{W}_{\mathrm{MHD}} / \mathrm{P}_{\mathrm{h}}$ are displayed as stars in figure 13. The expected slight power degradation of the confinement can be seen from the relation between $\mathrm{P}_{\mathrm{h}}$ and $\mathrm{W}_{\mathrm{MHD}}$, shown in figure 13 as insert.

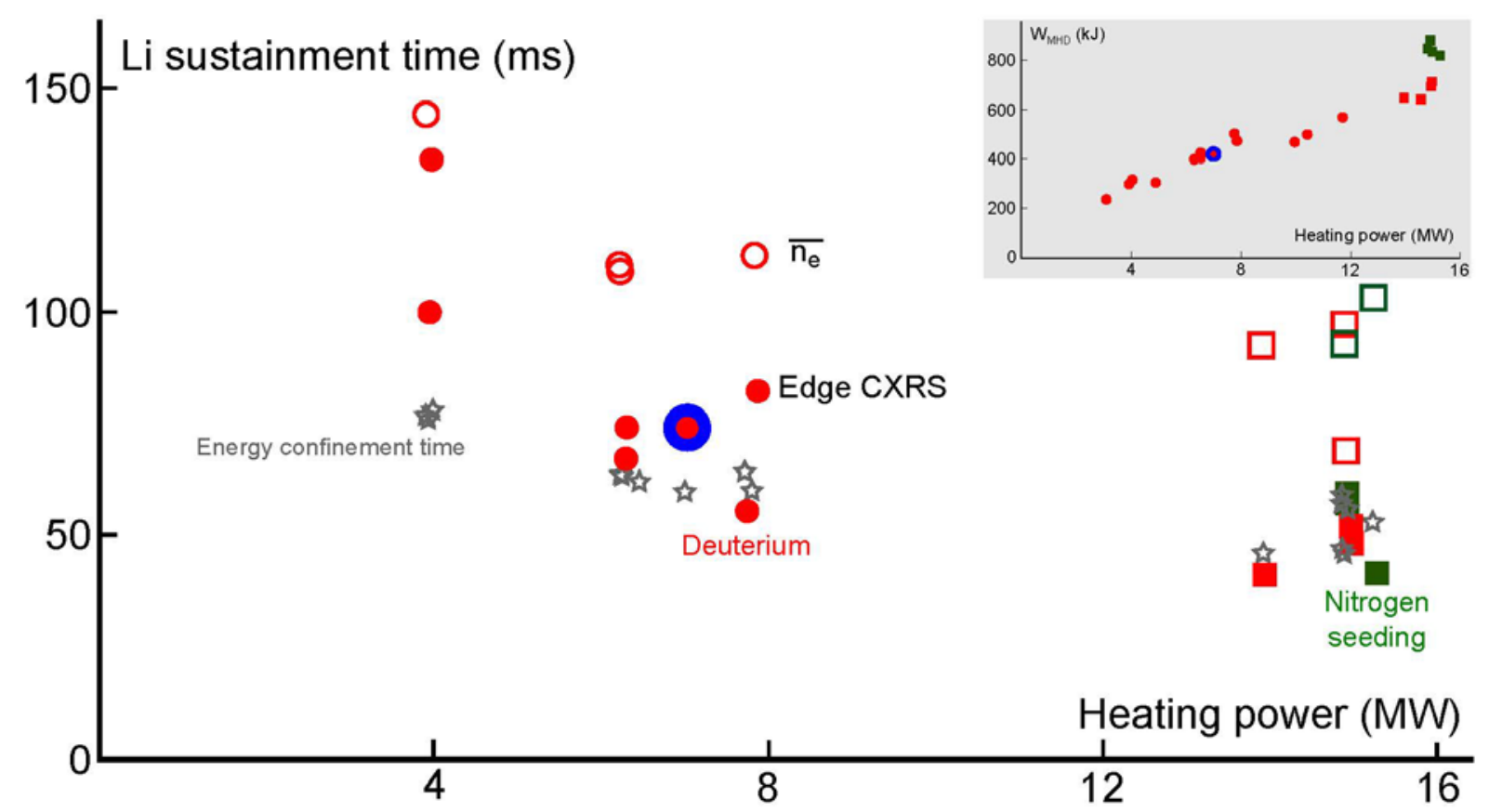

Figure 13: Li sustainment time versus $\mathrm{P}_{\mathrm{h}}$ determined from the evolution of $\overline{\mathrm{n}_{\mathrm{e}}}$ (open symbols) and the CXRS radiation from the edge (filled symbols). Colours and symbols as in figure 12 . Stars: energy confinement time approximated by $\mathrm{W}_{\mathrm{MHD}} / \mathrm{P}_{\mathrm{h}}$. The relation $\mathrm{W}_{\mathrm{MHD}}$ versus $\mathrm{P}_{\mathrm{h}}$ is shown in the grey insert.

\section{Li deposition on plasma facing components}

Although this investigation essentially aimed on finding out how $\mathrm{Li}$ in the core plasma impacts on the performance, we analysed as well if there is a possible influence via wall conditioning. Of course a strong interaction via Li deposition to PFCs is not expected since small Li amounts were used. Nevertheless, some features were found, caused by Li adhering transiently at the wall.

\subsection{Transient wall conditioning}

Following the introduction of $\mathrm{Li}$ via pellet injection, the presence of $\mathrm{Li}$ on PFCs could be detected in the monitoring signal of the Li beam diagnostic. Even after the Li density in the plasma decayed away, short spikes of Li induced radiation could be detected, correlated with the appearance of ELMs. This observation is attributed to the release of wall deposited Li by the energy impinging via the ELM, then transiently present in the edge plasma region. Although problematic for the intended profile analysis of the diagnostics, this behaviour allowed evaluation of the Li amount present on PFCs.

In this section, we consider an ensemble of discharges performed during the restart phase of the campaign mainly for testing and commissioning of vessel and heating systems with parameters $\mathrm{I}_{\mathrm{P}}=0.8 \mathrm{MA} ; \mathrm{B}_{\mathrm{t}}=2.46 \mathrm{~T} ; \mathrm{q}_{95}=5.0 ; \mathrm{V}_{\mathrm{p}}=12.5 \mathrm{~m}^{3} ; \kappa=1.78 ; \delta^{\mathrm{u}}=0.12$ and 
$\delta^{l}=0.45$. All but one of these discharges took place before the first vessel boronization. The first one is \#31895, a reference before any injection of Li pellets. Pellet injection was performed at $2 \mathrm{~Hz}$ in \#31898 launching 2 pellets and in \#31899 launching 5 pellets (shown in figure 2). The following discharges had no more pellets injected, three of them - \#31900, \#31902 and \#31904 - were selected for monitoring the Li disappearance. The concluding monitoring discharge \#31910 was performed shortly after vessel wall boronization. The analysis of these 7 shots was used to visualize deposition and erosion of Li on the PFCs and the according wall conditioning effect.

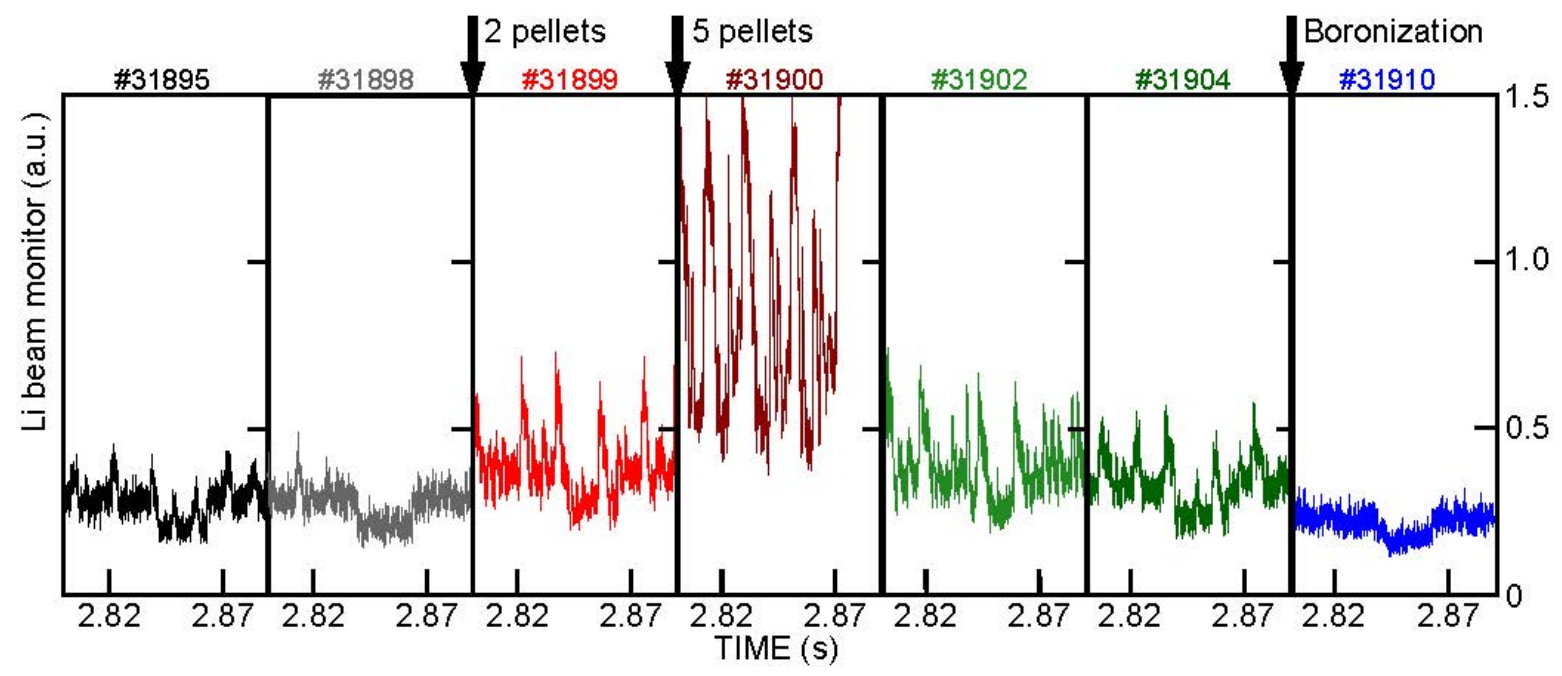

Figure 14: Li monitoring signal during an ELMy reference phase for a series of 7 discharges visualizing the deposition and erosion of Li introduced within 2 discharges comprising 7 pellets. The first vessel boronization took place shortly before the last discharge of the ensemble.

How the Li is deposited, present and eroded on the PFCs can be seen in figure 14, showing a short reference phase with ELM activity for every discharge of the ensemble. Since this chosen reference time interval is situated before pellet injection, the first two cases provide the nil reference before pellet actuation with still unconditioned walls. In discharge \#31899 the impact of 2 pellets can be observed; clearly visible short peaks correlating with the ELMs indicate a yet moderate but obvious presence of Li. The effect of all the seven pellets applied can be seen in discharge \#31900, representing the maximum of Li deposition. Notably, under such conditions Li beam data become too contaminated for a proper analysis to derive edge density and temperature profiles. Once the delivery of $\mathrm{Li}$ is stopped, the Li release from the wall vanishes quite quickly, as can be concluded from both following reference discharges. After boronization, no further $\mathrm{Li}$ is released. In addition, clean wall conditions result in a low impurity induced background signal. We infer from this observed evolution that a Li layer was deposited on PFCs after pellet injection, with a lifetime of only a few discharges. 


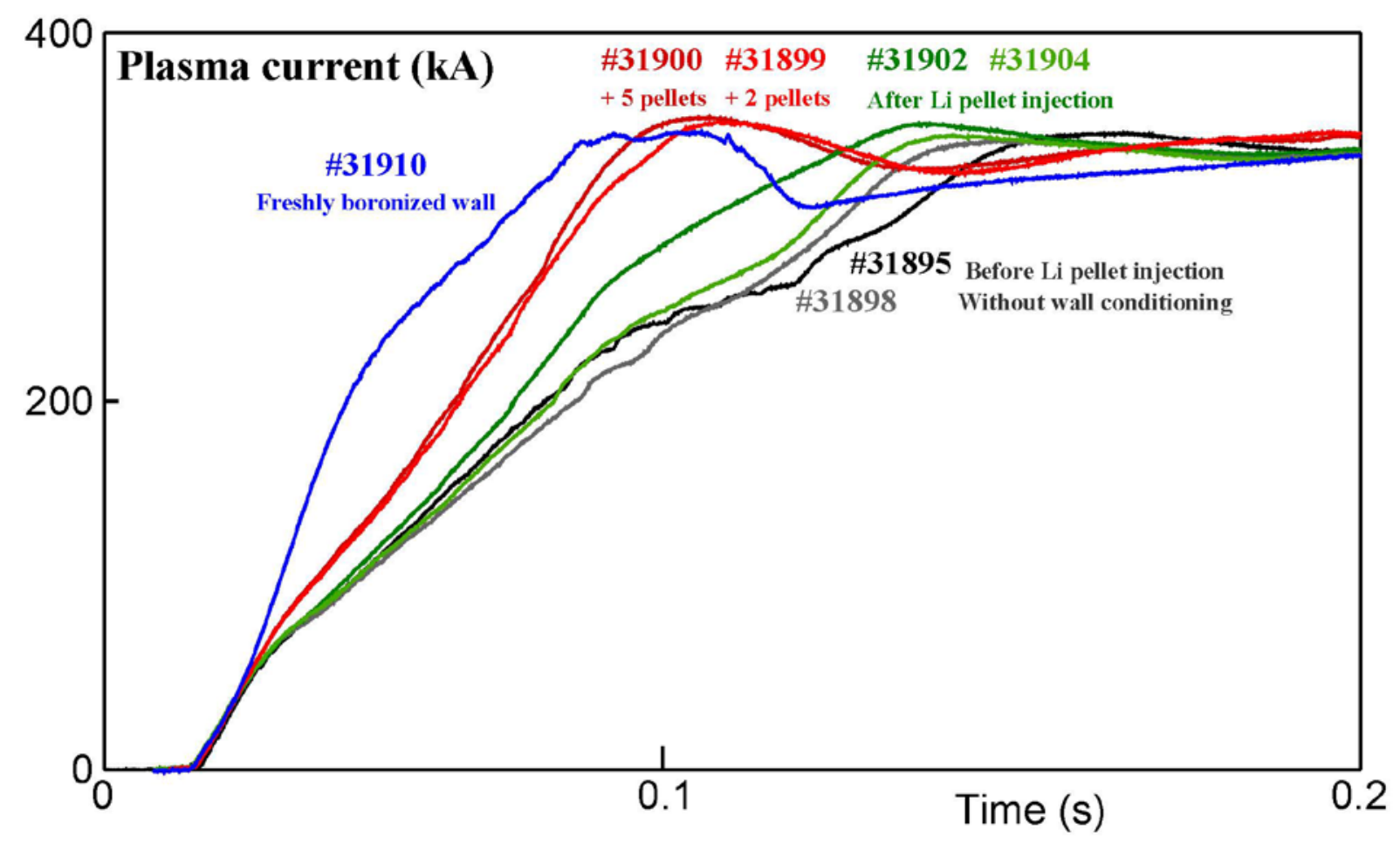

Figure 15: Plasma current ramp up for the analysed ensemble of discharges. Li pellets do improve wall conditions, the impact however seems to saturate already after 1-2 pellets, vanishes after few discharges and is smaller as the one resulting from a vessel boronization.

This interpretation is in full agreement with the small but clearly visible observed effect of the $\mathrm{Li}$ wall conditioning. It is well known that plasma operation can be improved by covering PFCs by a thin film of e.g. silicon, boron or $\mathrm{Li}$, e.g. by reducing impurity release from the wall [29]. This is apparently also the case here, as cleaner plasma conditions allow for an easier current ramp up, reducing the inductive flux consumption. Figure 15 shows the evolution of the plasma current in the early ramp up phase after break down for all discharges of the ensemble. As long as the $\mathrm{Li}$ or later $\mathrm{B}$ coverage is present, a faster ramp up as a signature of improved wall conditions can be observed. For the two cases before any wall conditioning and Li injection, a rather slow ramp up is observed. The situation is improved by the Li deposition; it is likely that the amount introduced by the initial 2 pellets has almost the same effect as compared with the full $2+5$ pellet sequence. Wall conditioning is however short-lived and quickly lost, as the ramp up in discharge \#32904 is almost back the initial condition. Li pellets can hence yield only a very temporary modest beneficial effect. As indicated by the final reference case with a B covered wall, the effect is more pronounced and it lasts for several operational days covering typically 25 discharges each.

\subsection{Persistent effect on sandblasted thermography tile}

From all the investigations it appears Li stays on PFCs only for a very short period, with a single exception. One of the vertical outer bulk W divertor tiles (poloidal position indicated by the filled black contour in Figure 3), in contact with the outer strike point in a typical lower single null configuration, is a special tile dedicated for thermography measurements of the power flux [30]. The surface of this tile installed in octant 5 has been sandblasted to increase 
the surface roughness and hence the IR emissivity, reducing direct reflections and to obtain a reference for movement detection. Here, a change of the emissivity was recognized after each $\mathrm{Li}$ pellet injection into plasmas. A reduction of the emissivity was observed at the strike line location. For all the discharges with $\mathrm{P}_{\mathrm{h}}$ up to about $12 \mathrm{MW}$ (before the monitoring experiment), the emissivity recovered within a few discharges. However, after the first monitoring discharge applying a total heating power of about $15 \mathrm{MW}$, the emissivity did not fully recover, even by the end of the entire campaign. This indicates an irreversible reaction of $\mathrm{Li}$ at such surfaces beyond a certain heat flux threshold.

Figure 16 shows the outer divertor target at 3 different stages. The left frame was recorded after the last discharge prior to the monitoring experiments. It is seen that the sandblasted area appears brighter than the surrounding area, and is more or less homogeneous in emission. The middle frame is recorded after the first discharge using Li pellets in combination with a significantly higher auxiliary heating power. The strike line position is indicated by the red ellipse. Now, a dark area is visible in the position where the strike line intersected the target. This suggests a decrease of the surface emissivity due to interaction of the target surface with the $\mathrm{Li}$ in the plasma. The right frame shows the same region after about 150 discharges without additional Li. The dark region at the strike line position is still visible. Due to the persistence of this reduced emissivity, it is concluded that the surface properties of the W target itself have been changed, and that the observed change is not caused due to deposition of $\mathrm{Li}$ on the target. 


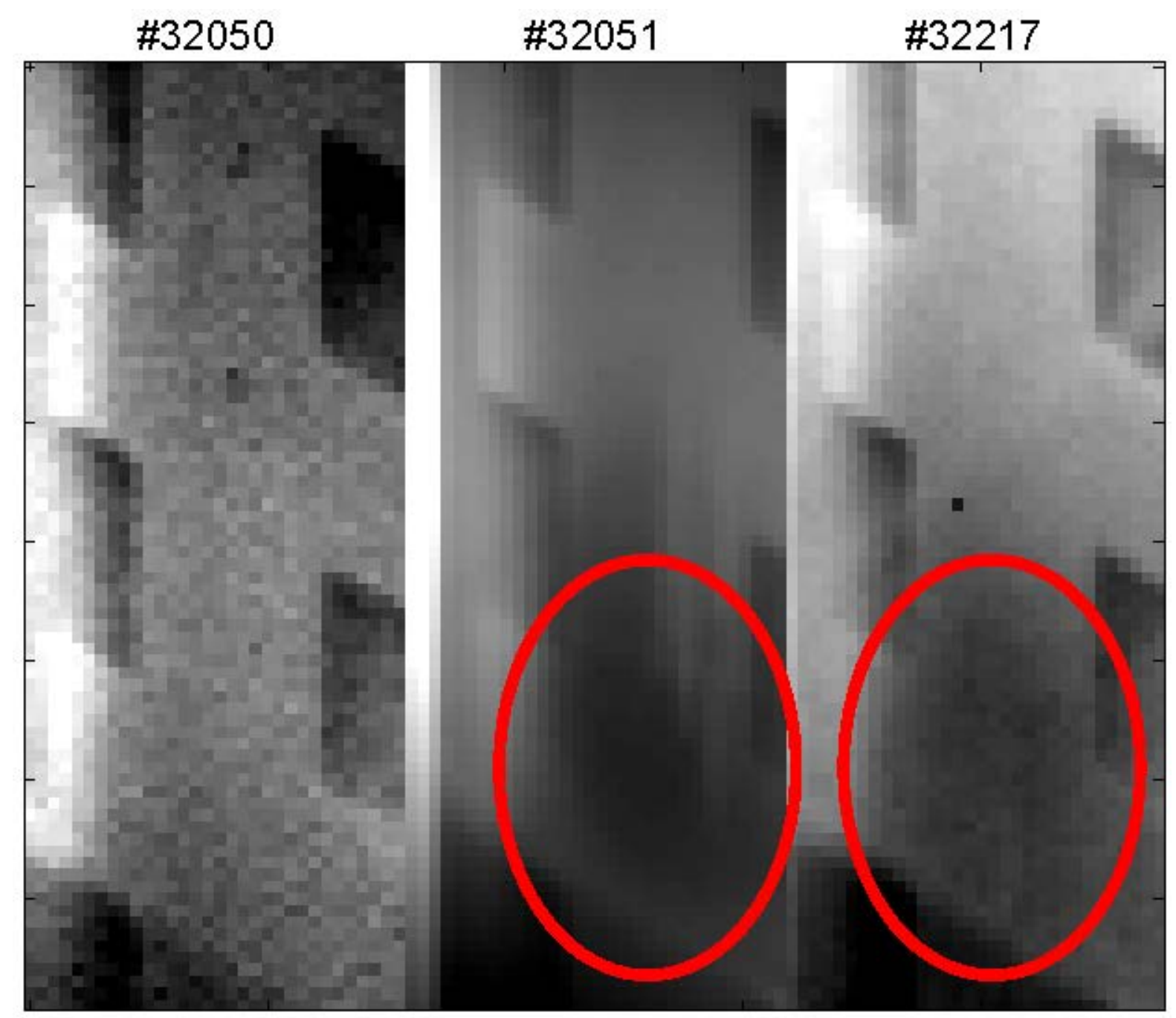

Figure 16: Sand blasted structure of the vertical outer bulk $W$ divertor tile inserted for thermography measurements (filled black contour in Figure 3). Frames taken at 3 different stages: After last discharge before (left) and first discharge with Li pellets into monitoring discharge with high $P_{h}$. Change of albedo is still lasting after 150 discharges run without Li injection (right).

\subsection{Post-mortem tile analysis}

Two standard bulk W vertical target tiles were mounted on the divertor manipulator (DIM-II), which is located in the outer divertor in octant 2 (filled black contour in figure 3). These tiles were exposed during the entire Li pellet experiments lasting several days and extracted shortly afterwards. One of these tiles was analysed ex-situ at the IPP tandem accelerator by means of nuclear reaction analysis (NRA). Spectra were recorded with D projectiles at $800 \mathrm{keV}$ exploiting the reaction ${ }^{7} \mathrm{Li}\left(\mathrm{D},{ }^{4} \mathrm{He}\right){ }^{5} \mathrm{He}$. A poloidal scan in steps of 5 or $10 \mathrm{~mm}$ was performed from the lower end of the tile, across the strike point to roughly $10 \mathrm{~cm}$ above. It was estimated that the detection limit is rather high at about $2 \times 10^{16} \mathrm{Li} / \mathrm{cm}^{2}$ due to some noise from other reactions. No clear $\mathrm{Li}$ assigned peak could be observed at any of the analysed poloidal positions. Thus we conclude that the $\mathrm{Li}$ concentration everywhere on the tile is below the detection limit. With a total of about $1.3 \times 10^{22} \mathrm{Li}$ atoms introduced, there is no obvious pileup in the divertor, otherwise a concentration $\sim 10^{18} \mathrm{~cm}^{-2}$ would have been observed. 
Assuming a homogeneous distribution on all plasma-wetted surfaces with a typical plasma surface of $42 \mathrm{~m}^{2}$, a Li concentration of about $3 \times 10^{16} \mathrm{~cm}^{-2}$ is expected, i.e. slightly above the detection limit (assuming a uniform density of the layer this would correspond to a thickness of roughly $6 \mathrm{~nm}$ ). Therefore, it seems the Li must have been partially exhausted and/or spread even to more remote areas.

The special sandblasted thermography tile showing the persistent change of the emissivity was dismounted after the end of the full experimental campaign. Still, a pronounced albedo change close to the strike line was visible. This tile was analysed too by means of NRA in order to clarify whether Li had been deposited onto the roughened surfaces. Close to the strike line position, spectra show several peaks indicating deposits. However, no Li signal is detected. Due to the increased background signal the detection limit for the area close to the strike line is about $1 \times 10^{16} \mathrm{Li} / \mathrm{cm}^{2}$. Three $\mathrm{cm}$ above the strike line NRA peaks and background are much smaller lowering the detection limit to about $4 \times 10^{15} \mathrm{Li} / \mathrm{cm}^{2}$; however again no indication for $\mathrm{Li}$.

\section{ELM trigger potential}

To master the challenge of ELMs is one of the major tasks in large fusion devices. For example, ITER will aim on ELM frequency control during the initial non-active low-current operation in order to keep $\mathrm{W}$ contamination of the plasma low and prevent excessive $\mathrm{W}$ core radiation losses. During the high current high fusion performance deuterium-tritium operation, mitigation of the power flux to the $\mathrm{W}$ divertor has to be achieved in order to prevent unacceptable damage [31]. Pellet pacing has been found a suitable candidate for these purposes. Injecting pellets produced from $\mathrm{D}$ fuel showed in a proof-of-principle experiment at AUG equipped still with a $\mathrm{C}$ wall the possibility to control the ELM frequency and even mitigate the power flux [32]. DIII-D demonstrated an enhancement of the spontaneous ELM frequency by a factor of 12 with an energy flux to the divertor mitigated accordingly [33]. In an all-metal-wall environment, however, it turned out more intricate to achieve ELM triggering by fuelling pellets. Neither at JET nor at AUG the capability for a completely reliable actuation was sustained after replacing the $\mathrm{C}$ wall. Instead, a scenario dependent lag time was observed; spontaneous or triggered ELM became possible only after this lag time [34]. Replacing small size fuelling pellets usually applied for ELM pacing by another suitable material like $\mathrm{Li}$ could be considered as a solution to resolve this issue, in particular with respect to the recently reported encouraging results using sub-mm Li granules into DIII-D plasmas [8].

In this study, it was not possible to perform dedicated ELM pacing experiments due to the restricted available pellet rate. In addition, pellet size and speed chosen cannot be regarded best suited for triggering investigations. Nevertheless, all available pellets have been analysed for their capability to trigger ELMs. For the low pellet rate and comparable short Li sustainment times, every pellet can be regarded as a single event that probes the stability of the plasma edge against the imposed perturbation. It was possible to identify three typical cases of response to pellet injection with respect to the trigger potential for sound type-I ELMs. An example for each of these three cases is shown in figure 17. It was taken care, that the pellet arrives in the plasma at a time when the occurrence of a spontaneous ELM is rather unlikely. In each case the upper box displays the thermo-current ELM monitor while the lower box shows both pellet ablation monitors. While the vertical view represents the magnitude of ablation and hence the perturbation strength, the horizontal view provides 
precise information about onset and end of the perturbation. Case one shown in the upper part of figure 17 documents a clear instant trigger of a type-I ELM An ELM similar to a type-I event is initiated by the pellet, and starts to evolve with the pellet ablation still in progress. Hence, it can be supposed the ELM is triggered directly by the local perturbation imposed by the pellet. Case two, middle part of the figure, shows a failed trigger event. In contrast to the previous case, no strong type-I ELM is taking place for several ms after injection. It shows a behaviour very likely to cases where D pellets fail to trigger ELMs. Instead the purely pellet born pressure pulse resulting from parts of the ablation plasmoid structure, which is partially expelled from the plasma follows the same dynamics as the ELM filament [35]. Such events observed for a few pellets found being incapable to impact on the ELM behaviour could challenge the claim for pellet pacing to serve a reliable tool for ELM control. The lower part of the figure displays case three; here an ELM is triggered, but only due to a change of the local plasma conditions. For such cases, the ELM onset starts $3-4$ ms delayed with respect to the ablation onset, but still at a time with negligible likelihood for a spontaneous ELM. At the time of the ELM onset, the strong local perturbation caused by the pellet has already homogenised its helical structure into a radially extending perturbation of the density and temperature profiles. This change of the profiles, transiently altering as well the power flux into the edge region, can trigger an ELM. Such behaviour has been already found when attempting to trigger an ELM by the impact of a pulsed supersonic D gas jet [36]. Due to the corresponding significant impact on the plasma parameters in the edge, enhancing the ELM frequency by such means appears unfavourable for a proper control approach.
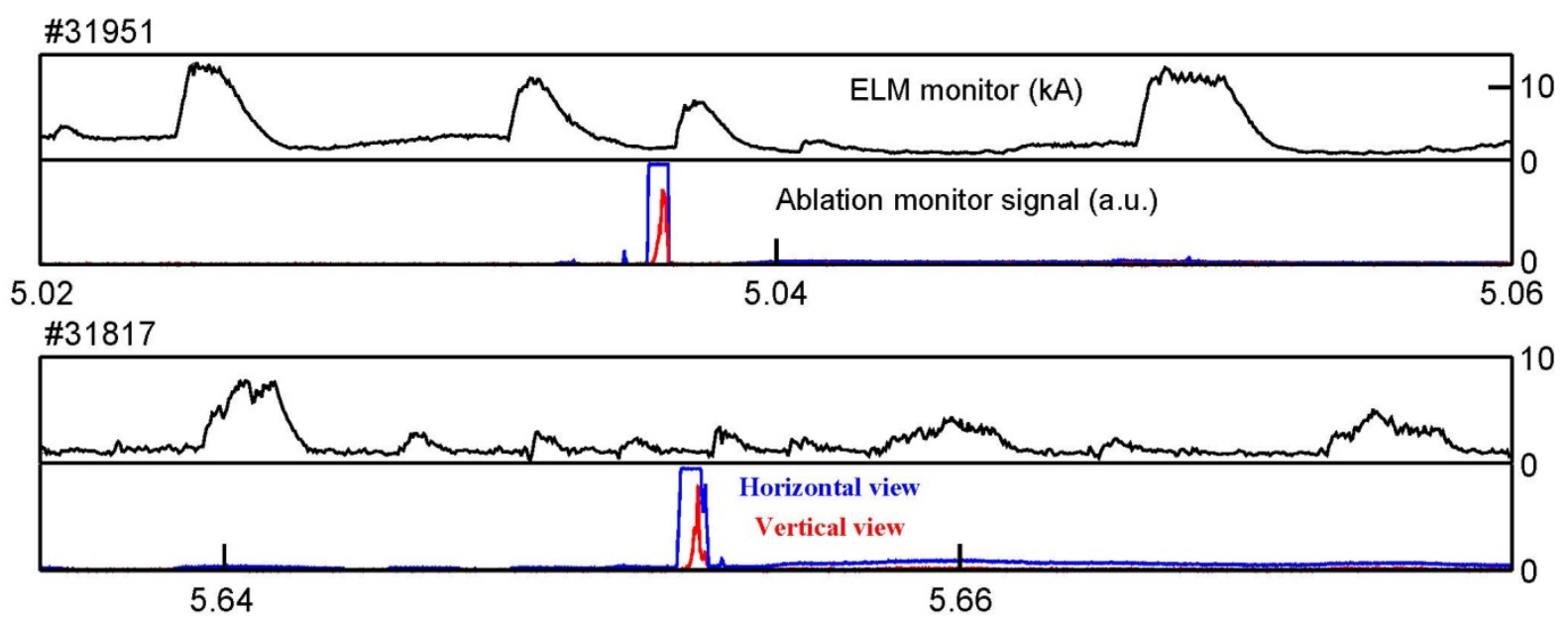

\#31899

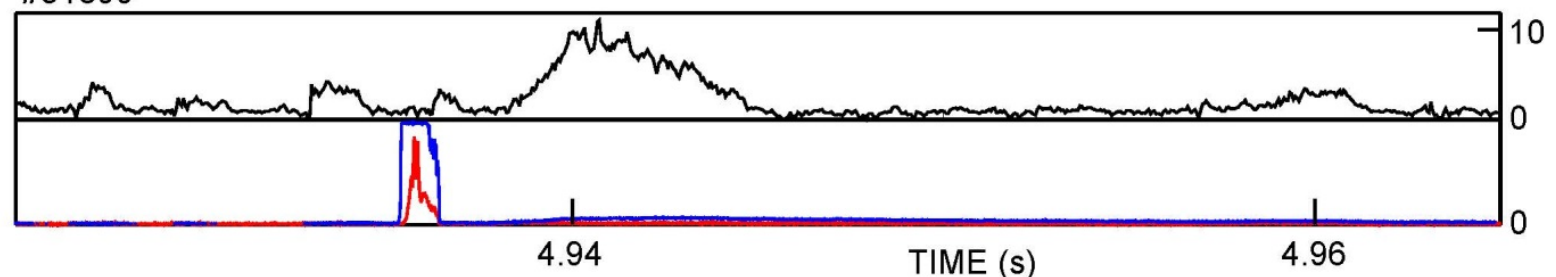

Figure 17: Typical cases observed for the response to a pellet with respect to ELM triggering. Immediate triggering (upper), no triggering (middle) and delayed indirect triggering (lower). For each case the divertor thermo-current ELM monitor (upper box) and both pellet ablation monitor signals (lower box) are plotted. 
Provided a sufficient dataset is available for a given scenario, the perturbative probing approach applying a small pellet rate is capable to determine if there is a lag time for type-I ELM triggering [34]. Unfortunately, the scarce amount of data, the diversity of target plasmas and many cases where pellets arrive in during high frequency ELM phases at a time a spontaneous ELM is likely to appear, did not allow for a proper statistical analysis. Nevertheless, some basic trends could be derived from the dataset showing instant triggering. Figure 18 shows the time delay between pellet ablation and triggered ELM onset times versus the time elapsed since the previous (spontaneous) ELM has occurred. Typically, the ELM starts to evolve about $0.06-0.18 \mathrm{~ms}$ after the pellet's crossing the separatrix. Very similar values have been found for D fuelling pellets where the ELM onset starts $0.1-0.2 \mathrm{~ms}$ after ablation onset [34]. ELMs triggered late in the ELM cycle tend to expel a larger fraction of the plasma energy than those triggered earlier in the ELM cycle.

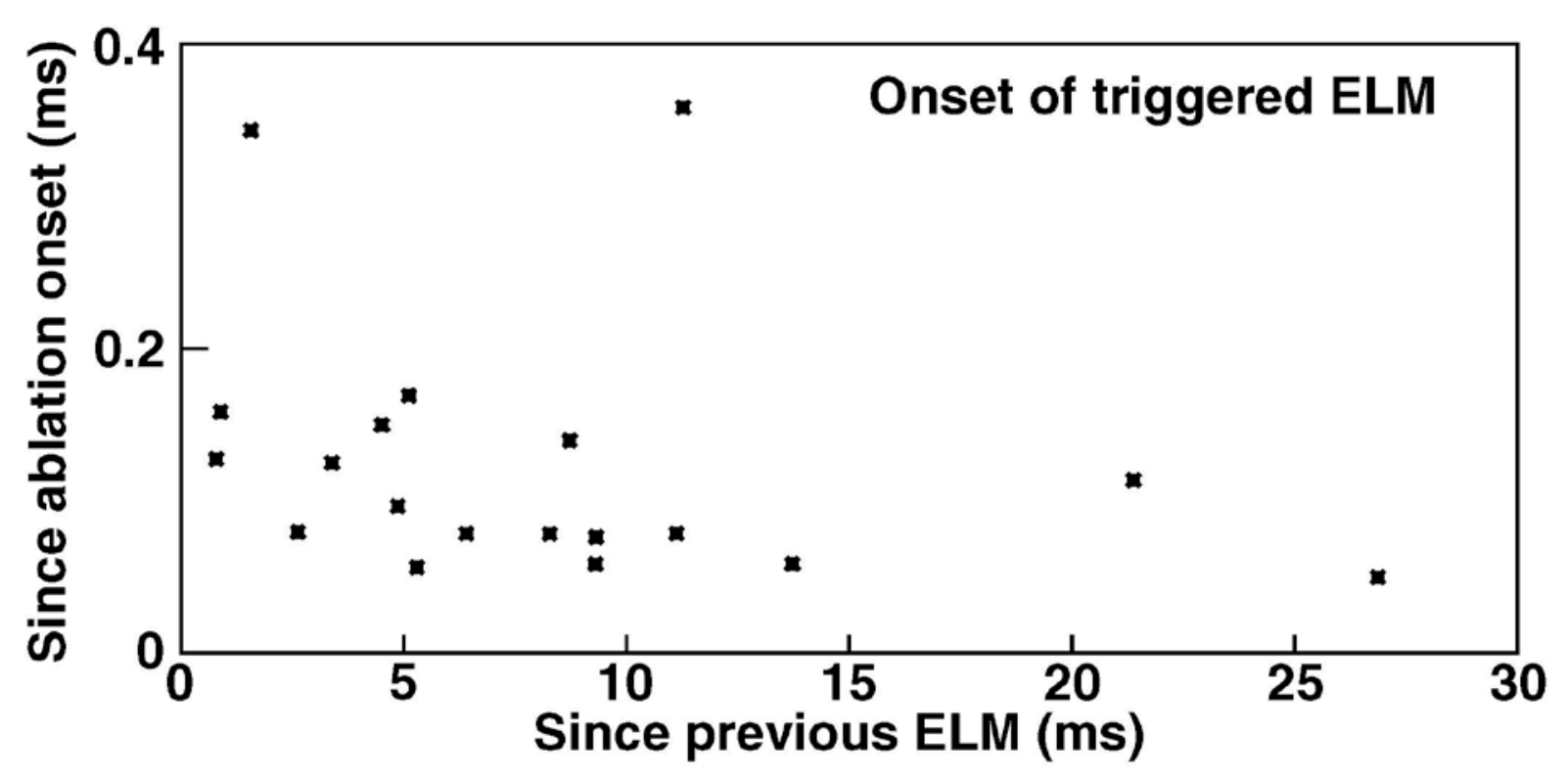

Figure 18: Time delay between onset of pellet ablation and triggered ELM versus time delay between triggered and preceding spontaneous ELM. All cases show instantly triggered ELMs ("case one" shown in figure 17).

Despite the limited set of data and unfavourable boundary conditions, it looks like the used kind of Li pellets is not perfectly suited for reliable ELM triggering. Possibly, a better adapted scheme employing smaller and slower pellets or granules imposing a larger local perturbation like demonstrated on EAST [37] and DIII-D [7] could yield a better reliability. Noteworthy, the task to find out if well-tailored $\mathrm{Li}$ pellets can provide reliable ELM control in a reactor relevant all-metal-wall configuration is still pending and worthwhile further dedicated investigations.

\section{Summary and outlook}

Experiments were performed at AUG to test the claim that $\mathrm{Li}$ can improve plasma performance in an all-metal-wall tokamak. For our explorative investigations, we took a fast and simple solution, employing a reconfigured gas gun. Thus, it was possible to inject an 
ample amount of Li deep into the plasma, avoiding early deposition on PFCs. As planned, a sufficiently high plasma concentration of $\mathrm{Li}$ was achieved, if only transiently. The $\mathrm{Li}$ sustainment times found range below $150 \mathrm{~ms}$; hence due to the low pellet injection rate of 2 $\mathrm{Hz}$, no pile-up in the plasma could take place. The impact of (the transient) Li on the plasma energy confinement was investigated in different scenarios. No indication was found that $\mathrm{Li}$ in the AUG plasma fosters an improved performance. Usually even a loss of up to about $5 \%$ of the plasma stored energy was observed. In scenarios where $\mathrm{N}$ seeding was applied for plasma energy confinement enhancement, an even more pronounced drop of plasma energy of up to about $7 \%$ took place, eliminating a significant fraction of the $\mathrm{N}$ induced enhancement. Additional radiation losses have been identified to cause the major part of the Li induced energy loss; longer Li sustainment times at lower heating power caused higher losses. In the $\mathrm{N}$ seeding scenario a fuelling induced effect contributes as well, causing an even more distinct energy drop. It cannot be ruled out, however, that the beneficial effects of Li seen in e.g. NSTX, EAST, and DIII-D were not observed in AUG either because of too little Li usage or because of the chosen transient delivery technique.

Investigations on the $\mathrm{Li}$ pellet ELM trigger potential have been possible only in the perturbative approach taking every single pellet for probing the edge stability; no dedicated frequency control and mitigation experiments could be performed. Seemingly, as has previously observed for fuelling pellets, large and fast Li pellets are like fuelling pellets in an all-metal-wall environment, i.e. incapable to grant a fully reliable ELM triggering.

Even small amounts of injected Li showed the potential for beneficial wall conditioning by cladding the PFCs. This has been manifested in the positive impact on the plasma formation before boronization, observed after the injection of only a few pellets. However, the effect is very short lived, as is the presence of the $\mathrm{Li}$ on PFCs. As a caveat the Li beam edge profile diagnostic is inoperable during this transient phase. A persisting alteration of a sand blasted $\mathrm{W}$ surface exposed to sufficiently high heat fluxes in the divertor has been realized. However, there is no Li pile up in the divertor, and most likely even no persistent presence of $\mathrm{Li}$ at all on the plasma-facing surfaces. Hence the change in the $\mathrm{W}$ surface was not due to formation of $\mathrm{Li}$ related compounds.

Because of the risk of a more severe wall contamination and the deleterious impact on the $\mathrm{Li}$ beam diagnostics, we refrained from follow-up experiments that would require larger amounts of $\mathrm{Li}$ via pellet or granules. The Li pellet injection system is now commissioned for the injection of room temperature solid state pellets without causing a major burden on the plasma by the propellant gas. Thus, the launcher might experience a revival for the injection of alternative material e.g. for the purpose of future wall conditioning experiments.

\section{Acknowledgment}

We are grateful to Dr. Garrard Conway for helpful dicussions. This work has been carried out within the framework of the EUROfusion Consortium and has received funding from the Euratom research and training programme 2014-2018 under grant agreement No 633053. The views and opinions expressed herein do not necessarily reflect those of the European Commission. 


\section{References}

[1] H.W. Kugel et al., Phys. Plasmas 15 (2008), 056118

[2] R. Maingi et al., Phys. Rev. Letts. 103 (2019) 075001

[3] R. Maingi et al., Phys. Rev. Letts. 107 (2011) 145004

[4] J.S. Hu et al., Phys. Rev. Lett. 114 (2014), 055001

[5] G.Z. Zuo, J.S. Hua, J.G. Li, Z. Su, D.K. Mansfield, L.E. Zakharov, Journal of Nuclear Materials 438 (2013), S90-S95

[6] ITER EXPERT GROUPS, et al, Nucl. Fusion 39 (1999), 2175

[7] T.H. Osborne et al., Nucl. Fusion 55 (2015), 063018

[8] A. Bortolon et al., Nucl. Fusion 56 (2016), 056008

[9] A. Herrmann et al., Nucl. Fusion 55 (2015), 063015

[10] R. Arredondo Parra et al., Rev. Sci. Instr. 87 (2016), 023508

[11] H.P. Summers, The ADAS User Manual, version 2.6 (2004), htttp://www.adas.ac.uk

[12] M. Kaufmann, K. Lackner, L. Lengyel, and W. Schneider, Nucl. Fusion 26 (1986), 171

[13] R. Fischer, C.J. Fuchs, B. Kurzan, W. Suttrop, E. Wolfrum, ASDEX Upgrade Team, Fusion Sci. Techn. 58 (2010), 675

[14] A. Kallenbach et al., Plasma Phys. Control. Fusion 52 (2010), 055002

[15] B. Pegourie, Plasma Phys. Control. Fusion 49 (2007) R87

[16] I. Erofeev et al., P1.037, EPS conference 2016

[17] E. Fable et al., Plasma Phys. Contr. Fusion 55, (2013), 12402

[18] G. M. Staebler et al., Phys. Plasmas 15 (2008), 055908

[19] R. Neu et al., Journal Nucl. Mat. 438 (2013), S34

[20] M. Beurskens et al., Nucl. Fusion 56 (2016), 056014

[21] R. Neu et al., 35th EPS Conference (2008), Europhysics Conference Abstracts ECA Vol. 32D, P-4.039

[22] A. Kappatou et al., 21 ${ }^{\text {st }}$ EU-US Transport Task Force Meeting, Leysin, September 2016

[23] S. Potzel et al., Journal of Nuclear Materials 463 (2015) 541

[24] M.G. Dunne et al., submitted to Plasma Phys. Control. Fusion

[25] R. Neu et al., Nucl. Fusion 45 (2005), 209

[26] R. Fischer, E. Wolfrum, J. Schweinzer, ASDEX Upgrade Team, Plasma Phys. Control

Fusion 50 (2008) 085009.

[27] P.T. Lang et al., P1.127, EPS conference 2015

[28] T. Pütterich, E. Fable, R. Dux, R. Neu, M.G. O’Mullane, R. Wenninger, P4.111, EPS conference 2015

[29] D. Naujoks, “Plasma-Material Interaction in Controlled Fusion”, Springer 2006, ISSN $1615-5653$

[30] B. Sieglin et al., Rev. Sci. Instrum. 86, 113502 (2015)

[31] A. Loarte et al., Nucl. Fusion 54 (2014), 033007

[32] P.T. Lang et al., Nucl. Fusion 44 (2004), 665

[33] L.R. Baylor et al., Phys. Rev. Lett. 110 (2013), 245001

[34] P.T. Lang et al., Nucl. Fusion 54 (2014), 083009

[35] P.T. Lang et al., Nucl. Fusion 51 (2011), 033010

[36] P.T. Lang et al., Plasma Phys. Control. Fusion 47 (2005) 1495

[37] D.K. Mansfield et al., Nucl. Fusion 53 (2013) 113023 